\title{
Inclusive jet production as a probe of polarized parton distribution functions at a future EIC
}

\author{
Radja Boughezal, ${ }^{1, *}$ Frank Petriello, ${ }^{1,2, \dagger}$ and Hongxi Xing ${ }^{1,2, \$}$ \\ ${ }^{1}$ High Energy Physics Division, Argonne National Laboratory, Argonne, Illinois 60439, USA \\ ${ }^{2}$ Department of Physics and Astronomy, Northwestern University, Evanston, Illinois 60208, USA
}

(Received 24 July 2018; published 27 September 2018)

\begin{abstract}
We present a detailed phenomenological study of polarized inclusive jet production in electron-proton collisions at a future Electron-Ion Collider (EIC). Our analysis is performed at next-to-leading order in perturbative QCD using the numerical code DISTRESS and includes all relevant partonic channels and resolved photon contributions. We elucidate the role of different kinematic regions in probing different aspects of proton and photon structure. We emphasize the importance of inclusive jet measurements and high collision energies at a future EIC in order to further constrain the polarized gluon distribution of the proton.
\end{abstract}

DOI: 10.1103/PhysRevD.98.054031

\section{INTRODUCTION}

Among the most intriguing aspects of hadronic physics is the spin decomposition of the proton in terms of its partonic constituents. This has remained an outstanding puzzle for three decades [1,2], and is one of the key motivations for the proposed Electron Ion Collider (EIC) [3]. This topic has attracted tremendous attention from both the experimental and theoretical QCD communities. To determine the contribution of quarks and gluons to the spin of the proton, according to the spin sum rule [4,5], one needs to extract the helicity-dependent parton distribution functions (PDFs) inside the proton. A standard way to approach this goal is to perform a global QCD analysis of all available data taken in spin-dependent deep inelastic scattering (DIS) and proton-proton ( $\mathrm{pp}$ ) collisions, such as is done by the de Florian-Sassot-Stratmann-Vogelsang (DSSV) [6] and NNPDF [7] collaborations. The accuracy of these global fits relies upon the validity of QCD factorization and the high precision computation of the perturbative hard coefficients.

Collimated jets of hadrons are one of the main probes of the unpolarized partonic structure of the nucleon in current global fits [8-10]. Due to the hard scale arising from the jet transverse momentum, there are small final-state

\footnotetext{
*rboughezal@anl.gov

†f-petriello@northwestern.edu

"hxing@northwestern.edu
}

Published by the American Physical Society under the terms of the Creative Commons Attribution 4.0 International license. Further distribution of this work must maintain attribution to the author(s) and the published article's title, journal citation, and DOI. Funded by SCOAP ${ }^{3}$. nonperturbative hadronic effects in inclusive jet measurements with large jet radius when the jet substructure is not probed. For the same reason, the double longitudinal spin asymmetry for jet production with large transverse momentum in DIS and pp collisions offer excellent sensitivity to the spin-dependent parton distribution functions of the individual quarks and gluons in the proton. In particular, a global analysis of the RHIC data [6] based on next-toleading order (NLO) perturbative QCD on jet production in polarized proton-proton collisions has revealed clear evidence for a significant contribution of gluon spin to the proton spin in the large momentum fraction region. However, information from low momentum fractions is still missing due to the lack of data in this region. With the center-of-mass energies available at the proposed EIC, polarized electron-proton runs will open unique possibilities to study jet production for a wide range of inclusive jet transverse momentum and rapidity, which in principle can provide access to the low momentum fraction region. Considering as well the anticipated high luminosity of the EIC, we anticipate that it can shed light on the helicitydependent parton distribution functions and provide a deeper understanding of the fundamental spin structure of the proton. Similar analyses have shown that the EIC can play an important role in understanding the nuclear dependence of PDFs [11,12].

The process of inclusive jet production in DIS without observing the outgoing lepton has been proposed to study the polarized PDFs in Ref. [13]. Recently, a NLO computation of the double longitudinal spin asymmetry in this process has been performed in Refs. [14,15], using the narrow cone approximation [16] to enable a fully analytical calculation. Previous work has studied aspects of inclusive jet photoproduction in polarized collisions $[17,18]$, within 
the framework of the subtraction method and narrow cone approximation, respectively. In all of these NLO studies $[14,15,17,18]$, opportunities to access the parton distributions of the polarized photon and proton are discussed. In this paper, we aim to provide a fully differential NLO Monte Carlo computation for inclusive jet production in DIS, which allows any kinematic cut to be imposed on the final state. In particular, we implement the recently developed $\mathrm{N}$-jettiness subtraction scheme $[19,20]$ extended to describe polarized collisions [21] in order to regularize all QCD infrared divergences. We present a detailed phenomenological analysis of inclusive jet production in polarized electron-proton scattering at a future EIC. We summarize below several key aspects and findings of our study.

(i) We study three possible collision energies for a future EIC to determine how they differ in their sensitivity to proton structure. Higher collision energies generally offer sensitivity to more aspects of hadronic structure, particularly to the hadronic structure of the proton.

(ii) We include all partonic contributions relevant to inclusive jet production, including both direct and resolved photon contributions that become relevant when the final-state lepton travels collinear to the beam direction. By including all relevant channels we are able to determine which regions of phase space are sensitive to different aspects of proton structure.

(iii) We perform a detailed study of the unpolarized cross section and double longitudinal spin asymmetry throughout the accessible kinematic range in transverse momentum and pseudorapidity, and point out which regions are sensitive to which aspects of proton structure. Particularly in higher-energy collisions, different regions of jet transverse momentum can be selected to probe either the resolved photon distributions or the helicity-dependent proton PDFs.

(iv) We estimate the effect of EIC statistical errors and current PDF errors on jet production. The PDF errors are much larger than the estimated statistical ones over much of phase space, demonstrating the EIC potential to greatly improve our knowledge of PDFs.

(v) We quantify the effect of changing the jet radius on EIC phenomenology, which turns out to be small. A primary finding of our study is that the observable $A_{L L}$ in inclusive jet production at high collision energies is very sensitive to the gluon helicity distribution, particularly in the high transverse momentum and forward rapidity region, thus providing a good channel to reduce the uncertainty in determining the gluon contribution to the proton spin. Our analysis is complementary to other important studies that have demonstrated the sensitivity of EIC jet production to various other aspects of proton structure [22-24], as described later in the text.

The rest of the paper is organized as follows. We review our fixed-order perturbative QCD theoretical framework in
Sec. II and detail all partonic channels included in our calculation. All relevant scattering processes have been incorporated into the numerical code DISTRESS [25]. In Sec. III we detail the numerical settings and parameter choices used in our study. Numerical results for the three chosen EIC collision energies are presented in Secs. IV-VI. Finally, we summarize and conclude in Sec. VII.

\section{THEORETICAL FRAMEWORK}

We sketch here the theoretical framework used in our study. For more details we refer the reader to the discussion in Ref. [25]. Our analysis is performed using fixed-order perturbative QCD through $\mathcal{O}\left(\alpha_{s}\right)$ in the strong coupling constant. We include the leading contributions in the electromagnetic coupling, which go as $\mathcal{O}\left(\alpha^{2}\right)$. We express the hadronic cross section in the following notation:

$$
\mathrm{d} \sigma=\mathrm{d} \sigma_{\mathrm{LO}}+\mathrm{d} \sigma_{\mathrm{NLO}}+\cdots,
$$

where the ellipsis denotes neglected higher-order terms. This generic equation holds for both the polarized and unpolarized cross sections. The LO subscript refers to the $\mathcal{O}\left(\alpha^{2}\right)$ term while the NLO subscript denotes the $\mathcal{O}\left(\alpha^{2} \alpha_{s}\right)$ correction. For the partonic cross sections, we introduce superscripts that denote the powers of both $\alpha$ and $\alpha_{s}$ that appear. For example, the leading quark-lepton scattering process is expanded as

$$
\mathrm{d} \hat{\sigma}_{q l}=\mathrm{d} \hat{\sigma}_{q l}^{(2,0)}+\mathrm{d} \hat{\sigma}_{q l}^{(2,1)}+\cdots .
$$

Here, the $\mathrm{d} \hat{\sigma}_{q l}^{(2,0)}$ denotes the $\mathcal{O}\left(\alpha^{2}\right)$ correction, while $\mathrm{d} \hat{\sigma}_{q l}^{(2,1)}$ indicates the $\mathcal{O}\left(\alpha^{2} \alpha_{s}\right)$ term. The leading-order (LO) hadronic cross section can be written as a convolution of parton distribution functions with these partonic cross sections,

$$
\begin{aligned}
\mathrm{d} \sigma_{\mathrm{LO}}= & \int \frac{\mathrm{d} \xi_{1}}{\xi_{1}} \frac{\mathrm{d} \xi_{2}}{\xi_{2}} \sum_{q}\left[f_{q / H}\left(\xi_{1}\right) f_{l / l}\left(\xi_{2}\right) \mathrm{d} \hat{\sigma}_{q l}^{(2,0)}\right. \\
& \left.+f_{\bar{q} / H}\left(\xi_{1}\right) f_{l / l}\left(\xi_{2}\right) \mathrm{d} \hat{\sigma}_{\bar{q} l}^{(2,0)}\right]
\end{aligned}
$$

Here, $f_{q / H}\left(\xi_{1}\right)$ is the usual parton distribution function that describes the distribution of a quark $q$ in the hadron $H$ carrying a fraction $\xi_{1}$ of the hadron momentum. $f_{l / l}\left(\xi_{2}\right)$ is the distribution for finding a lepton with momentum fraction $\xi_{2}$ inside the original lepton. At leading order this is just $f_{l / l}\left(\xi_{2}\right)=\delta\left(1-\xi_{2}\right)$, but it is modified at higher orders in the electromagnetic coupling by photon emission. The dependence of these distribution functions on the $\overline{\mathrm{MS}}$ factorization scale $\mu_{F}$ is implicit. $d \hat{\sigma}_{q l}^{(2,0)}$ is the differential partonic cross section. At leading order only the partonic channel $q\left(p_{1}\right)+l\left(p_{2}\right) \rightarrow q\left(p_{3}\right)+l\left(p_{4}\right)$ and the same process with antiquarks contributes. The relevant Feynman diagram is shown in Fig. 1. 


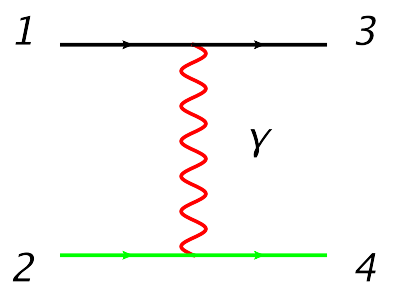

FIG. 1. Feynman diagram for the leading-order process $q\left(p_{1}\right)+l\left(p_{2}\right) \rightarrow q\left(p_{3}\right)+l\left(p_{4}\right)$. We have colored the photon line red, the lepton lines green and the quark lines black.

At the next-to-leading order level several new structures appear. The leading-order quark-lepton scattering channel receives both virtual and real-emission corrections that are separately infrared divergent. We have performed two calculations using dipole subtraction [26] and $N$-jettiness subtraction $[19,20]$ to regularize and cancel these divergences. The agreement we find between these two approaches serves as a check of our result. Initial-state collinear divergences are absorbed into PDFs via mass factorization. At this order in perturbation theory a gluonlepton scattering channel also contributes. The collinear divergences that appear in this channel are removed by mass factorization. Representative Feynman diagrams for these processes are shown in Fig. 2.

We can express the NLO hadronic cross section in the following form:

$$
\begin{aligned}
\mathrm{d} \sigma_{\mathrm{NLO}}= & \int \frac{\mathrm{d} \xi_{1}}{\xi_{1}} \frac{\mathrm{d} \xi_{2}}{\xi_{2}}\left\{f_{g / H}^{1} f_{l / l}^{2} \mathrm{~d} \hat{\sigma}_{g l}^{(2,1)}+f_{g / H}^{1} f_{\gamma / l}^{2} \mathrm{~d} \hat{\sigma}_{g \gamma}^{(1,1)}\right. \\
& \left.+\sum_{i=q, \bar{q}}\left[f_{i / H}^{1} f_{l / l}^{2} \mathrm{~d} \hat{\sigma}_{i l}^{(2,1)}+f_{i / H}^{1} f_{\gamma / l}^{2} \mathrm{~d} \hat{\sigma}_{i \gamma}^{(1,1)}\right]\right\},
\end{aligned}
$$

where we have abbreviated $f_{i / j}^{k}=f_{i / j}\left(\xi_{k}\right)$. The contributions $\mathrm{d} \hat{\sigma}_{g l}^{(2,1)}, \mathrm{d} \hat{\sigma}_{q l}^{(2,1)}$ and $\mathrm{d} \hat{\sigma}_{\bar{q} l}^{(2,1)}$ denote the usual DIS partonic channels computed to NLO in QCD with zero lepton mass. The terms $\mathrm{d} \hat{\sigma}_{q \gamma}^{(1,1)} \mathrm{d} \hat{\sigma}_{\overline{q \gamma}}^{(2,1)}$ and $\mathrm{d} \hat{\sigma}_{g \gamma}^{(1,1)}$ denote new contributions arising when $Q^{2}=-\left(p_{2}-p_{4}\right)^{2} \approx 0$. These are associated with a virtual photon that is nearly

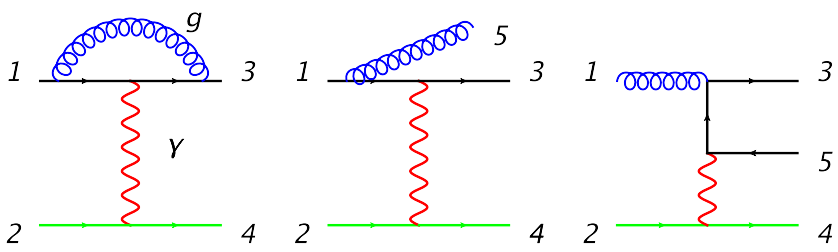

FIG. 2. Representative Feynman diagrams contributing to the perturbative QCD corrections at NLO: virtual corrections to the $q\left(p_{1}\right)+l\left(p_{2}\right) \rightarrow q\left(p_{3}\right)+l\left(p_{4}\right)$ process (left), real emission correction $q\left(p_{1}\right)+l\left(p_{2}\right) \rightarrow q\left(p_{3}\right)+l\left(p_{4}\right)+g\left(p_{5}\right) \quad$ (middle), and the process $g\left(p_{1}\right)+l\left(p_{2}\right) \rightarrow q\left(p_{3}\right)+l\left(p_{4}\right)+\bar{q}\left(p_{5}\right)$ (right). We have colored the photon line red, the lepton lines green, the gluon lines blue and the quark lines black.

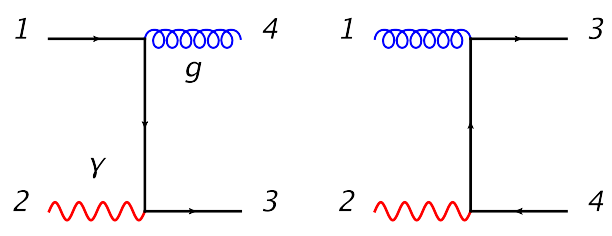

FIG. 3. Representative Feynman diagrams contributing to the $q\left(p_{1}\right)+\gamma\left(p_{2}\right) \rightarrow q\left(p_{3}\right)+g\left(p_{4}\right) \quad($ left $)$ and $g\left(p_{1}\right)+\gamma\left(p_{2}\right) \rightarrow$ $q\left(p_{3}\right)+\bar{q}\left(p_{4}\right)$ scattering processes.

on shell, and a final-state lepton that travels down the beam pipe. The transverse momentum of the leading jet is balanced by the additional jet present in these diagrams, and the final-state lepton is not observed. This kinematic configuration leads to a QED collinear divergence for vanishing lepton mass. While it is physically regulated by the lepton mass, it is more convenient to obtain these corrections by introducing a photon distribution function in analogy with the usual parton distribution function. The collinear divergences that appear in the matrix elements computed with vanishing lepton mass can be absorbed into this distribution function. This procedure is described for inclusive jet production at Born level in Ref. [13], and at higher orders in Refs. [25,27]. Representative diagrams for the photon-initiated processes are shown in Fig. 3.

Since it is our primary calculational tool we give here a brief description of the $N$-jettiness subtraction technique as introduced in Ref. [19]. The starting point of this method is the $N$-jettiness event shape variable [28], defined in the one-jet case of current interest as

$$
\mathcal{T}_{1}=\frac{2}{Q^{2}} \sum_{i} \min \left\{p_{B} \cdot q_{i}, p_{J} \cdot q_{i}\right\}
$$

Here, $p_{B}$ and $p_{J}$ are lightlike four-vectors along the initialstate hadronic beam and final-state jet directions, respectively. This definition of the event shape variable $\mathcal{T}_{1}$ in Eq. (5) is dimensionless, and corresponds to $\tau_{1}^{a}$ in Ref. [29]. The $q_{i}$ denote the four-momenta of all final-state partons. Values of $\mathcal{T}_{1}$ near 0 indicate a final state containing a single narrow energy deposition, while larger values denote a final state containing two or more well-separated energy depositions. Restricting $\mathcal{T}_{1}>0$ removes all singular limits of the quark-lepton matrix elements, e.g., when the additional parton that appears in the real emission corrections is soft or collinear to the beam or the final-state jet. This can be seen from Eq. (5); if $\mathcal{T}_{1}>0$ then $q_{i}$ must be resolved. Since all unresolved limits are removed, the $\mathcal{O}\left(\alpha^{2} \alpha_{s}\right)$ correction in this phase space region can be obtained from a leadingorder calculation of two-jet production in electron-nucleon collisions. When $\mathcal{T}_{1}$ is smaller than any other hard scale in the problem, it can be resummed to all orders in perturbation theory [29-33]. Expansion of this resummation formula to $\mathcal{O}\left(\alpha^{2} \alpha_{s}\right)$ gives the NLO correction to the quark-lepton scattering channel for small $\mathcal{T}_{1}$. 
To obtain the full NLO result using this idea we partition the phase space into regions above and below a cutoff on $\mathcal{T}_{1}$, which we label $\mathcal{T}_{1}^{\text {cut }}$,

$$
\begin{aligned}
\mathrm{d} \sigma_{q l}^{(2,1)}= & \int \mathrm{d} \Phi_{\mathrm{V}}\left|\mathcal{M}_{\mathrm{V}}\right|^{2}+\int \mathrm{d} \Phi_{\mathrm{R}}\left|\mathcal{M}_{\mathrm{R}}\right|^{2} \theta_{1}^{<} \\
& +\int \mathrm{d} \Phi_{\mathrm{R}}\left|\mathcal{M}_{\mathrm{R}}\right|^{2} \theta_{1}^{>} \\
\equiv & \mathrm{d} \sigma_{q l}^{(2,1)}\left(\mathcal{T}_{1}<\mathcal{T}_{1}^{\text {cut }}\right)+\mathrm{d} \sigma_{q l}^{(2,1)}\left(\mathcal{T}_{1}>\mathcal{T}_{1}^{\text {cut }}\right) .
\end{aligned}
$$

We have abbreviated $\theta_{1}^{<}=\theta\left(\mathcal{T}_{1}^{\text {cut }}-\mathcal{T}_{1}\right)$ and $\theta_{1}^{>}=\theta\left(\mathcal{T}_{1}-\right.$ $\left.\mathcal{T}_{1}^{\text {cut }}\right)$ and have used the notation $\mathrm{R}$ and $\mathrm{V}$ to denote real and virtual corrections to the cross section. The first two terms in this expression have $\mathcal{T}_{1}<\mathcal{T}_{1}^{\text {cut }}$, and have been collectively denoted as $\mathrm{d} \sigma_{q l}^{(2,1)}\left(\mathcal{T}_{1}<\mathcal{T}_{1}^{\text {cut }}\right)$. The remaining term has $\mathcal{T}_{1}>\mathcal{T}_{1}^{\text {cut }}$, and has been denoted as $\mathrm{d} \sigma_{q l}^{(2,1)}\left(\mathcal{T}_{1}>\mathcal{T}_{1}^{\text {cut }}\right)$. We obtain $\mathrm{d} \sigma_{q l}^{(2,1)}\left(\mathcal{T}_{1}>\mathcal{T}_{1}^{\text {cut }}\right)$ from a LO calculation of two-jet production. This is possible since no unresolved limit occurs in this phase-space region. We derive $\mathrm{d} \sigma_{q l}^{(2,1)}\left(\mathcal{T}_{1}<\mathcal{T}_{1}^{\text {cut }}\right)$ using the all-orders resummation of this process [30,31].

Finally, nearly on-shell photons can also lead to resolved photon contributions to the cross section, in which these photons split into QCD partons that enter the hardscattering process. Since this splitting occurs for low virtualities this process receives important nonperturbative QCD contributions. Although the hard scattering corrections are formally $\mathcal{O}\left(\alpha_{s}^{2}\right)$, they can be sizeable due to the nonperturbative distribution of the partons inside the photon. We write these distributions as $f_{a / \gamma}(x, \mu)$ and $\Delta f_{a / \gamma}(x, \mu)$ in the unpolarized and polarized cases respectively. This leads to corresponding nonperturbative parton-in-lepton distributions. In the unpolarized case we have

$$
f_{a / l}(x, \mu)=\int_{x}^{1} \frac{d y}{y} P_{\gamma l}(y, \mu) f_{a / \gamma}\left(\frac{x}{y}, \mu\right) .
$$

The function $P_{\gamma l}$ in the leading-logarithmic approximation is given by

$$
P_{\gamma l}(y, \mu)=\frac{\alpha}{2 \pi} \frac{1+(1-y)^{2}}{y}\left[\ln \frac{\mu^{2}}{y^{2} m_{l}^{2}}-1\right] .
$$

In the polarized case these formulas take the form

$$
\begin{aligned}
\Delta f_{a / l}(x, \mu) & =\int_{x}^{1} \frac{d y}{y} \Delta P_{\gamma l}(y, \mu) \Delta f_{a / \gamma}\left(\frac{x}{y}, \mu\right), \\
\Delta P_{\gamma l}(y, \mu) & =\frac{\alpha}{2 \pi} \frac{2-y}{y}\left[\ln \frac{\mu^{2}}{y^{2} m_{l}^{2}}\right] .
\end{aligned}
$$

The form of the nonperturbative polarized parton distributions of the photon have not been determined from data and require modeling, as discussed in Ref. [34]. We can write the resolved-photon contribution to the unpolarized cross section as

$$
\begin{aligned}
\mathrm{d} \sigma_{\mathrm{res}}= & \int \frac{\mathrm{d} \xi_{1} \mathrm{~d} \xi_{2}}{\xi_{1} \xi_{2}}\left\{\sum_{i=q, \bar{q}}\left[f_{g / H}^{1} f_{i / l}^{2} \mathrm{~d} \hat{\sigma}_{g i}^{(0,2)}+f_{i / H}^{1} f_{g / l}^{2} \mathrm{~d} \hat{\sigma}_{i g}^{(0,2)}\right]+f_{g / H}^{1} f_{g / l}^{2} \mathrm{~d} \hat{\sigma}_{g g}^{(0,2)}\right. \\
& +\sum_{q}\left[f_{q / H}^{1} f_{\bar{q} / l}^{2} \mathrm{~d} \hat{\sigma}_{q \bar{q}}^{(0,2)}+f_{\bar{q} / H}^{1} f_{q / l}^{2} \mathrm{~d} \hat{\sigma}_{\bar{q} q}^{(0,2)}+f_{q / H}^{1} f_{q / l}^{2} \mathrm{~d} \hat{\sigma}_{q q}^{(0,2)}+f_{\bar{q} / H}^{1} f_{\bar{q} / l}^{2} \mathrm{~d} \hat{\sigma}_{\bar{q} \bar{q}}^{(0,2)}\right] \\
& \left.+\sum_{q \neq q^{\prime}}\left[f_{q / H}^{1} f_{q^{\prime} / l}^{2} \mathrm{~d} \hat{\sigma}_{q q^{\prime}}^{(0,2)}+f_{\bar{q} / H}^{1} f_{\bar{q}^{\prime} / l}^{2} \mathrm{~d} \hat{\sigma}_{\bar{q} \bar{q}^{\prime}}^{(0,2)}+f_{q / H}^{1} f_{\bar{q}^{\prime} / l}^{2} \mathrm{~d} \hat{\sigma}_{q \bar{q}^{\prime}}^{(0,2)}+f_{\bar{q} / H}^{1} f_{q^{\prime} / l}^{2} \mathrm{~d} \hat{\sigma}_{\bar{q} q^{\prime}}^{(0,2)}\right]\right\},
\end{aligned}
$$

where we have implicitly set the arguments of $f_{i / H}^{1}\left(\xi_{1}, \mu\right)=$ $f_{i / H}^{1}$ and $f_{i / l}^{2}\left(\xi_{2}, \mu\right)=f_{i / l}^{2}$. A similar expression holds in the polarized case. Although the partonic scattering cross sections go like $\mathcal{O}\left(\alpha_{s}^{2}\right)$ as the superscripts in Eq. (10) indicate, it can be shown that in certain limits the $f_{i / \gamma}$ distributions go like $1 / \alpha_{s}$ [35]. This leads to $f_{i / l} \propto$ $1 / \alpha_{s}$ through the dependence of $f_{i / l}$ on $f_{i / \gamma}$ contained in Eq. (7). We consequently count this contribution as part of the next-to-leading order result. Representative diagrams contributing to the partonic scattering processes of Eq. (10) are shown in Fig. 4. The total cross section becomes

$$
\mathrm{d} \sigma_{\mathrm{tot}}=\mathrm{d} \sigma_{\mathrm{LO}}+\mathrm{d} \sigma_{\mathrm{NLO}}+\mathrm{d} \sigma_{\text {res }} .
$$

All of these partonic channels have been incorporated into the numerical program DISTRESS [25], which predicts both polarized and unpolarized cross sections for jet production in DIS. A summary of the partonic
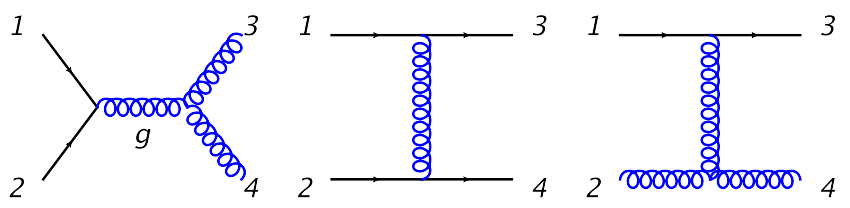

FIG. 4. Representative Feynman diagrams contributing to the $q\left(p_{1}\right)+\bar{q}\left(p_{2}\right) \rightarrow g\left(p_{3}\right)+g\left(p_{4}\right)$ (left), $q\left(p_{1}\right)+q^{\prime}\left(p_{2}\right) \rightarrow q\left(p_{3}\right)+$ $q^{\prime}\left(p_{4}\right)$ (middle), and $q\left(p_{1}\right)+g\left(p_{2}\right) \rightarrow q\left(p_{3}\right)+g\left(p_{4}\right)$ (right) scattering processes. 
TABLE I. Summary of partonic channels, the region of photon virtuality $Q^{2}$ for which they contribute, and the distribution functions to which they are sensitive.

\begin{tabular}{lcl}
\hline \hline $\begin{array}{l}\text { Partonic } \\
\text { channel }\end{array}$ & $Q^{2}$ region & \multicolumn{1}{c}{ Contributing PDFs } \\
\hline$q l$ & $Q^{2}>0$ & $f_{q / H}, \Delta f_{q / H}$ \\
$g l$ & $Q^{2}>0$ & $f_{g / H}, \Delta f_{g / H}$ \\
$q \gamma$ & $Q^{2} \approx 0$ & $f_{q / H}, f_{\gamma / l}, \Delta f_{q / H}, \Delta f_{\gamma / l}$ \\
$g \gamma$ & $Q^{2} \approx 0$ & $f_{g / H}, f_{\gamma / l}, \Delta f_{g / H}, \Delta f_{\gamma / l}$ \\
$q q$ & $Q^{2} \approx 0$ & $f_{q / H}, f_{q / \gamma}, \Delta f_{q / H}, \Delta f_{q / \gamma}$ \\
$q g$ & $Q^{2} \approx 0$ & $f_{q / H}, f_{q / \gamma}, \Delta f_{q / H}, \Delta f_{q / \gamma}, f_{g / H}, f_{g / \gamma}$, \\
& & $\Delta f_{g / H}, \Delta f_{g / \gamma}$ \\
$g g$ & $Q^{2} \approx 0$ & $f_{g / H}, f_{g / \gamma}, \Delta f_{g / H}, \Delta f_{g / \gamma}$ \\
\hline \hline
\end{tabular}

contributions in DISTRESS used in this analysis, the kinematic range in which they contribute, and the PDFs to which they are sensitive are shown in Table I.

\section{NUMERICAL SETUP}

We describe in this section the numerical parameters and settings used in our study. For convenience we summarize below in Table II the various kinematic quantities used in our analysis. We reconstruct jets using the anti- $k_{T}$ algorithm [36]. Unless noted otherwise we use a jet radius $R=0.8$. The transverse momenta and pseudorapidities of the jets are reconstructed in the center-of-mass frame of the electron-proton scattering process. We set the renormalization and factorization scales to the transverse momentum of the jet, $\mu_{R}=\mu_{F}=p_{T}^{j}$. A detailed study of the theoretical uncertainty arising from scale variation of the cross section was performed previously [25], with the conclusion that once the next-to-next-to-leading order (NNLO) corrections are incorporated the scale dependence is reduced to the few-percent level. Since we do not expect this to be a limiting uncertainty by the time of EIC datataking we do not consider the scale dependence further here.

For the unpolarized parton distributions in the proton we use the NNPDF 3.1 PDFs [10] extracted at next-toleading order in QCD perturbation theory. To describe

TABLE II. Definition of kinematic parameters used in our study.

\begin{tabular}{ll}
\hline \hline$\sqrt{s}$ & $\begin{array}{l}\text { Center-of-mass energy of the proton-lepton } \\
\text { collision }\end{array}$ \\
$Q^{2}=-\left(p_{2}-p_{4}\right)^{2}$ & $\begin{array}{l}\text { Virtuality of the photon exchanged in the } \\
\text { DIS process } \\
\text { Tranverse momentum of the observed jet in } \\
\text { the lab frame } \\
p_{T}^{j}\end{array}$ \\
$\eta^{j}$ & $\begin{array}{l}\text { Pseudorapidity of the observed jet in the } \\
\text { lab frame }\end{array}$ \\
\hline \hline
\end{tabular}

TABLE III. Considered run scenarios for a future EIC including center-of-mass scattering energies, jet transverse momentum ranges and jet pseudorapidity ranges.

\begin{tabular}{llc}
\hline \hline$\sqrt{s}$ & \multicolumn{1}{c}{$p_{T}^{\text {jet }}$ range } & $\eta^{j}$ et range \\
\hline $141.4 \mathrm{GeV}$ & $5 \mathrm{GeV} \leq p_{T}^{j} \leq 35 \mathrm{GeV}$ & $-3 \leq \eta^{j} \leq 3$ \\
$63.2 \mathrm{GeV}$ & $5 \mathrm{GeV} \leq p_{T}^{j} \leq 30 \mathrm{GeV}$ & $-2.5 \leq \eta^{j} \leq 2.5$ \\
$44.7 \mathrm{GeV}$ & $5 \mathrm{GeV} \leq p_{T}^{j} \leq 20 \mathrm{GeV}$ & $-2 \leq \eta^{j} \leq 2$ \\
\hline \hline
\end{tabular}

the polarized parton content of the proton we use the NNPDFpol1.1 polarized PDFs [7] unless noted otherwise. The one-sigma PDF uncertainties shown in the following sections are obtained by evaluating the cross section for the 100 replica sets provided by NNPDF and combining their differences from the reference set according to the standard methodology [37]. For the nonperturbative unpolarized parton distributions of the photon we use the leading-order Gluck-Reya-Vogt distributions from Ref. [38]. The corresponding polarized distributions have not been determined from data and require modeling, as discussed in Ref. [34]. We study both the minimal and maximal models from this reference, which correspond to different choices for the boundary conditions used when solving the evolution equations which these distributions satisfy. ${ }^{1}$ We note that all considered PDFs are defined in the $\overline{\mathrm{MS}}$ factorization scheme.

In order to estimate the sensitivity of inclusive jet production to the polarized structure of the proton for different EIC realizations, we consider three different setups corresponding to different center-of-mass scattering energies [39]. These different energies, together with the associated ranges of jet transverse momenta and pseudorapidities considered, are shown in Table III. We assume $10 \mathrm{fb}^{-1}$ of integrated luminosity for all design parameters. For simplicity we also assume $100 \%$ polarization for both the initial-state electrons and protons. The results we obtain can be simply rescaled to account for the polarization fractions eventually realized. To illustrate graphically what inclusive jet production at an EIC teaches us about proton structure, we show in Fig. 5 how the different measured $\left(p_{T}^{j}, \eta^{j}\right)$ regions map into the Bjorken- $x$ and $Q^{2}$ ranges of the PDFs. We assume leading-order $2 \rightarrow 2$ kinematics in order to make these plots. We see that particularly at high center-of-mass energies relatively low $\left(\propto 10^{-3}\right)$ Bjorken- $x$ can be probed.

We note that the experimental cuts considered allow for $Q^{2} \approx 0$, where the final-state lepton goes down the beam pipe and is not observed. This kinematic configuration allows for on-shell photons and is responsible for the parton-photon and parton-parton scattering channels that

\footnotetext{
${ }^{1}$ We thank W. Vogelsang for providing numerical routines for the polarized photon distributions.
} 


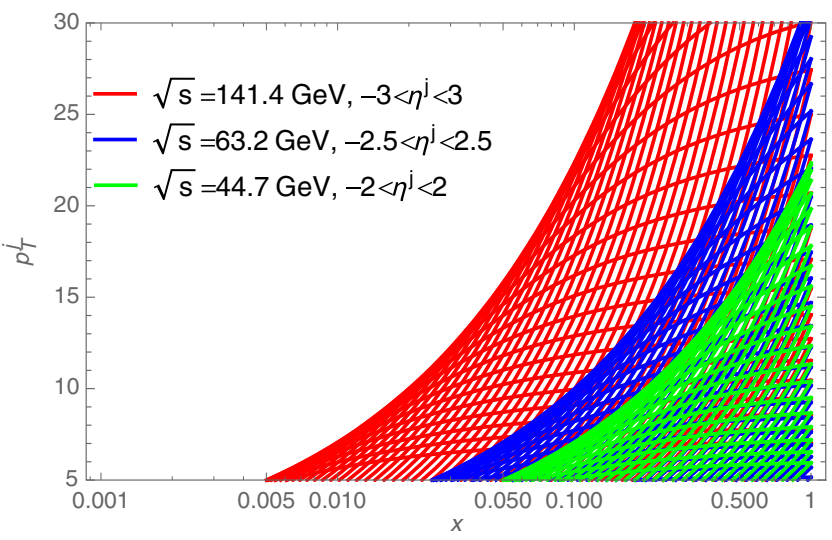

FIG. 5. Ranges of Bjorken- $x$ and $Q^{2}$ probed by inclusive jet measurements for each studied scattering energy.

appear in the previous section. In order to investigate the effects of these channels we also consider the effect of a $Q^{2}>10 \mathrm{GeV}^{2}$ cuts for the energy $\sqrt{s}=141.4 \mathrm{GeV}$. Such a cut removes the on-shell photon contributions.

\section{RESULTS FOR $\sqrt{s}=141.4 \mathrm{GeV}$}

We begin by presenting numerical results for the largest center-of-mass energy considered in our study, $\sqrt{s}=141.4 \mathrm{GeV}$. For this setting and for all other numerical results we consider both the total unpolarized cross section and the double-longitudinal spin asymmetry defined in Eq. (12). We study both quantities as functions of the jet transverse momentum and pseudorapidity. Both inclusive jet production without and with a tagged lepton are considered for this collider energy.
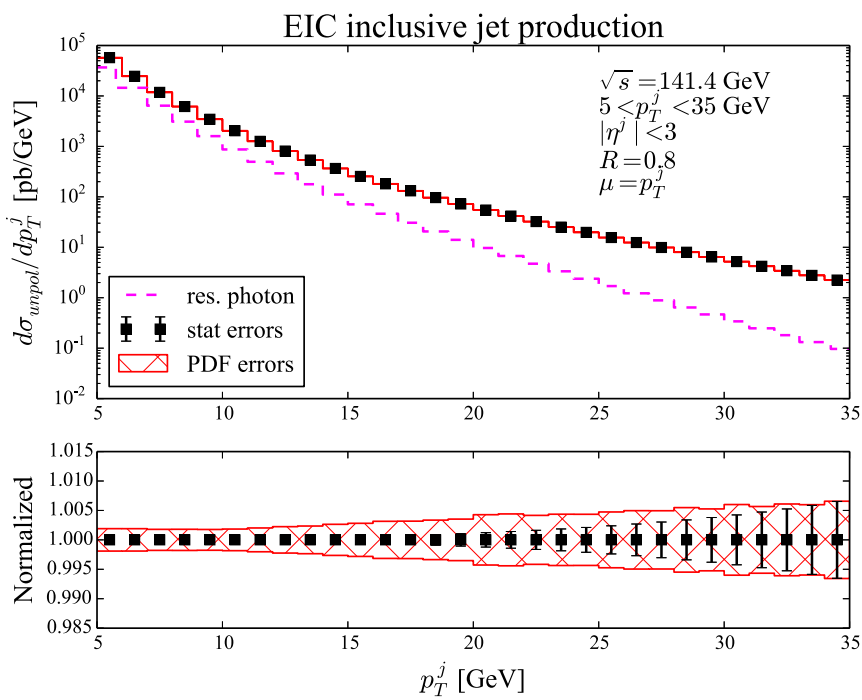

\section{A. Inclusive jet production without a tagged lepton}

We begin by presenting the unpolarized cross section as a function of both the jet transverse momentum and pseudorapidity in Fig. 6. No cut on the momentum transfer $Q^{2}$ is imposed, so that nearly on-shell photons contribute to the measured jet distributions. The red bands in these plots show the PDF uncertainties as computed using the NNPDF 3.1 error sets. Also shown are the statistical errors as represented by vertical bars on the black points estimated at a future EIC assuming $10 \mathrm{fb}^{-1}$ of integrated luminosity. The contributions to the cross section coming from resolved photons are shown separately in these plots as dashed lines. In the lower panels the results are normalized to the central value of the predictions in order to more clearly illustrate the errors. We see that the estimated PDF uncertainties are quite small, at or below the $1 \%$ level over most of the accessible kinematics. This is not surprising, as the unpolarized PDFs have been very well determined from a combination of HERA, LHC and lower energy data. The estimated experimental statistical errors assuming $10 \mathrm{fb}^{-1}$ of integrated luminosity are also at or below the $1 \%$ level except at high $p_{T}^{j}$ or in the high pseudorapidity regions. We note that we have not attempted to estimate the experimental systematic errors in our study. The resolved photon contribution to the cross section is important at low transverse momentum. It falls off rapidly as $p_{T}^{j}$ is increased, suggesting that determinations of this quantity should focus on the low transverse momentum region to enhance its importance as compared to other partonic contributions.

To make the partonic structure of these results more clear we show in Fig. 7 the central values for each distribution split into the separate partonic channels. The labeling of the
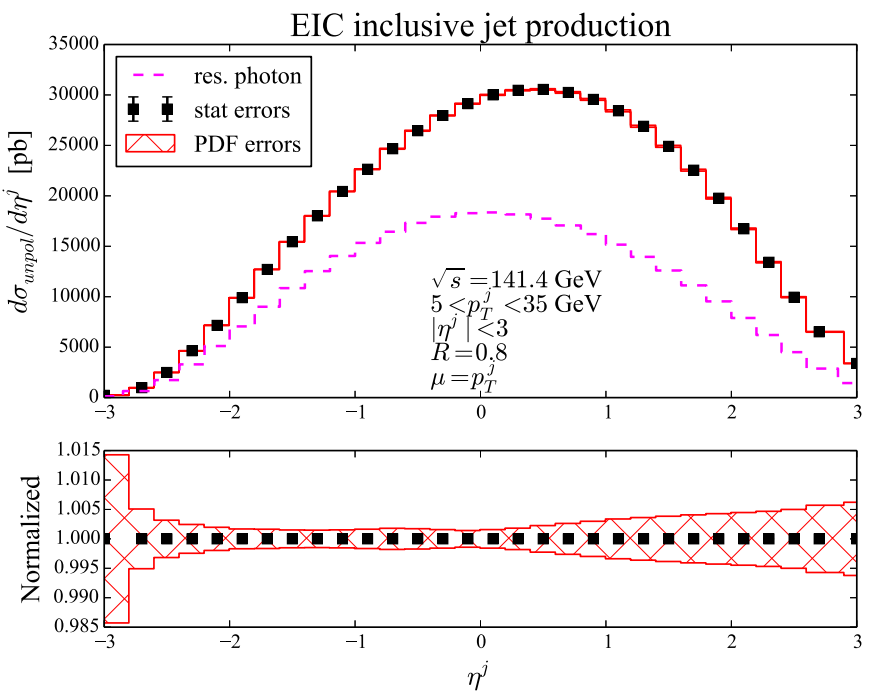

FIG. 6. Total unpolarized cross section as a function of jet transverse momentum (left panel) and jet pseudorapidity (right panel). The resolved photon contribution is shown separately in the upper panel of each plot. The lower panels normalize the results to the central values in order to more clearly illustrate the errors. 

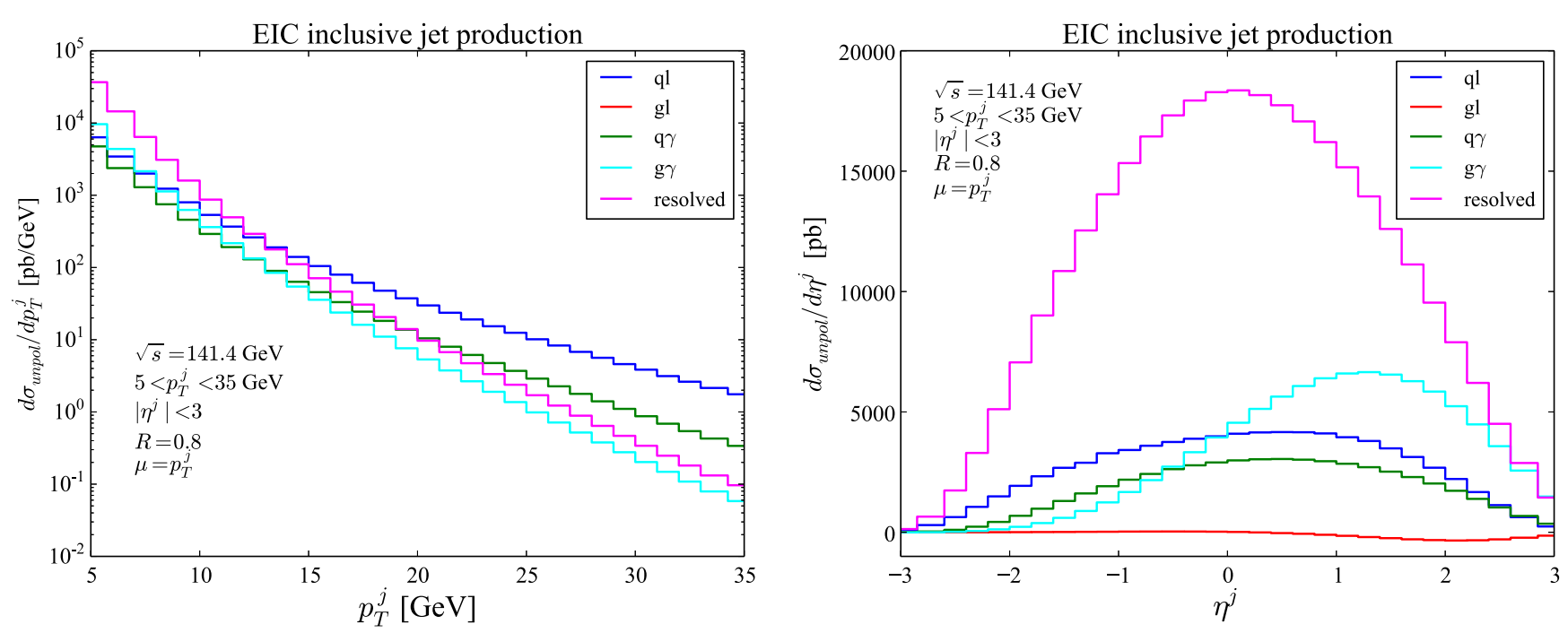

FIG. 7. Split of the unpolarized transverse momentum and pseudorapidity distributions into partonic channels as described in the text.

partonic channels follows that introduced in Sec. II: the $q l$ and $g l$ channels denote diagrammatic contributions of the form shown in Figs. 1 and 2 where the virtual photon exchanged between the quark and lepton line is off shell, $q \gamma$ and $g \gamma$ denote contributions of the sort shown in Fig. 3 in which the photon is nearly on shell and directly interacts with a parton coming from the proton, and the resolved contribution denotes terms where the photon splits at low virtuality into a parton before entering the hard scattering, as shown in Fig. 4. We first discuss the structure of the transverse momentum distribution. The resolved photon contributions dominate at low $p_{T}^{j}$ and fall off rapidly as $p_{T}^{j}$ is increased. This occurs because of the multiple collinear splittings needed to obtain the parton from the initial lepton as shown in Eq. (7), leading to softer distributions for $f_{q / l}$ and $f_{g / l}$. At intermediate values $p_{T}^{j} \sim 15-20 \mathrm{GeV}$ both the $q l$ and resolved channels are important, while at high $p_{T}^{j}$ the $q l$ channel dominates. The $q \gamma$ and $g \gamma$ channels are smaller than the leading channel for all $p_{T}^{j}$. The $g l$ channel is negligible throughout phase space. The $\eta^{j}$ distribution is dominated throughout phase space by the resolved photon channel. This is not surprising as the total event rate is dominated by low $p_{T}^{j}$ where this channel is largest. We note that the $g \gamma$ channel becomes important at high $\eta^{j}$. The sensitivity of the unpolarized jet production cross section to the resolved photon structure has been studied previously [23], where the possibility of flavor tagging to resolve the quark and gluon distributions has also been discussed.

We now study the double-longitudinal spin asymmetry, defined as

$$
\mathcal{A}_{L L}=\frac{d \sigma^{++}-d \sigma^{+-}-d \sigma^{-+}+d \sigma^{--}}{d \sigma^{++}+d \sigma^{+-}+d \sigma^{-+}+d \sigma^{--}}
$$

where the first superscript refers to the helicity of the lepton and the second one to the proton. The possibility of polarized beams at an EIC makes the measurement of this observable possible, allowing access to the polarized structure of the proton. The spin asymmetry as a function of jet transverse momentum and jet pseudorapidity is shown in Fig. 8. We again show the PDF errors and statistical errors for each distribution. The PDF errors are significantly larger than in the unpolarized case, indicating the poorer understanding of the polarized structure of the proton. The asymmetry increases as a function of $p_{T}^{j}$, reaching nearly $20 \%$ for $p_{T}^{j}=35 \mathrm{GeV}$. It is small throughout the studied $\eta^{j}$ range, since the event rate when integrated over $p_{T}^{j}$ is dominated by low transverse momentum where $A_{L L}$ is small. The fact that the PDF errors are much larger than the estimated statistical errors over all of phase space shows that the EIC has the potential to greatly improve our knowledge of these distributions. The increase of the PDF errors at low transverse momentum is due to the large uncertainty in the polarized PDFs at low Bjorken- $x$. Notice that the black points in Fig. 8 correspond to the ratio of central values of polarized and unpolarized cross sections from 100 replicas provided by NNPDF, which is different with the central values from 100 sets of $A_{L L}$. This explains why the black points are not encompassed by the red band as shown in the right plot of Fig. 8.

We show three additional quantities in the upper panels of each plot. First, we show the resolved photon contribution to the asymmetry for both the minimal and maximal models of the polarized distribution functions of the photon defined in Ref. [34]. We define these contributions by keeping only the resolved photon terms in the numerator of Eq. (12), while keeping all contributions to the denominator. The resulting quantity is directly proportional to the polarized photon distribution function. Both models of the resolved photon distribution give small contributions to 

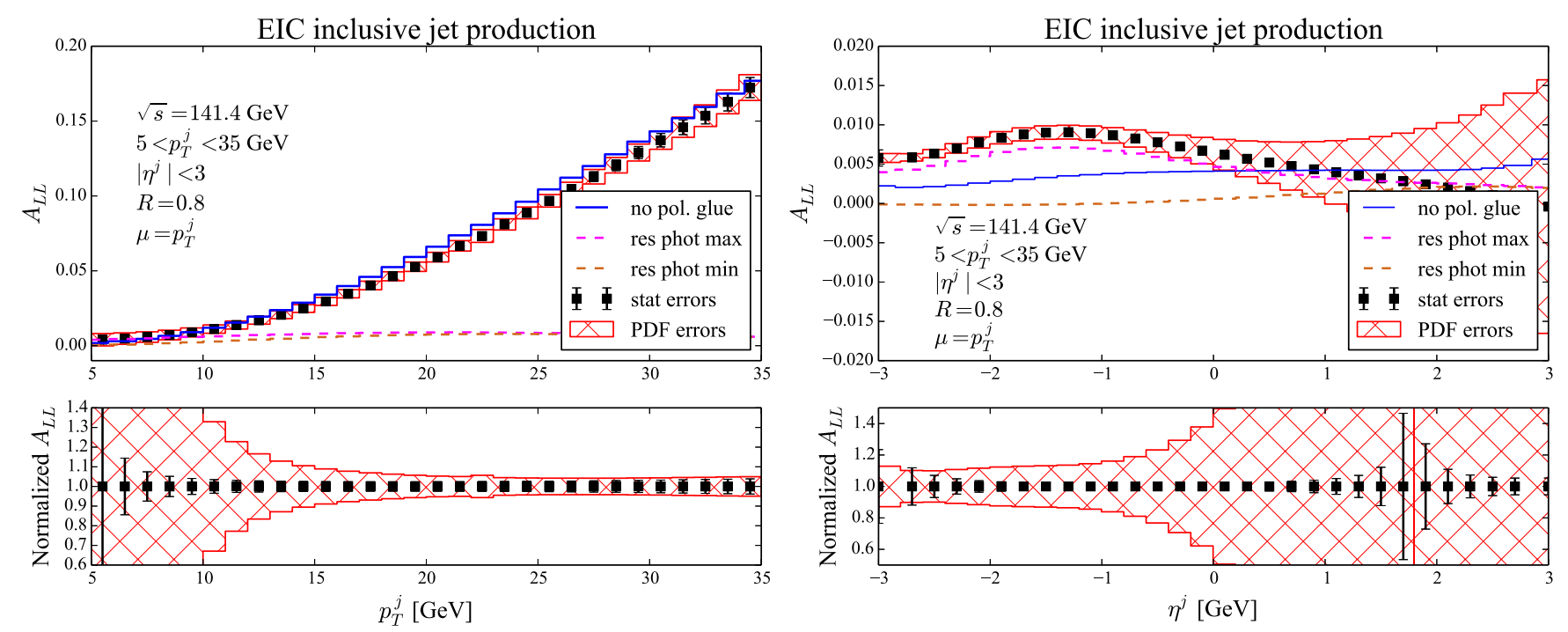

FIG. 8. Spin asymmetry as a function of jet transverse momentum (left panel) and jet pseudorapidity (right panel). The resolved photon contribution is shown separately in the upper panel of each plot. The lower panels normalize the results to the central values in order to more clearly illustrate the errors.

the asymmetry except at low values of $p_{T}^{j}$, indicating that intermediate and high- $p_{T}^{j}$ jet production is not sensitive to this distribution. The situation is different for the $\eta^{j}$ distribution. The maximal model gives nearly all of the asymmetry in the negative $\eta^{j}$ region, while the minimal model is small throughout the entire $\eta^{j}$ range. This indicates that the uncertainties arising from the polarized photon distributions are large in the $\eta^{j}$ distribution.

We also show in these plots the results where the polarized gluon distribution is set to 0 . These are obtained by setting all numerator terms containing the gluon distribution to 0 in Eq. (12), while keeping all contributions in the denominator. As the determination of the polarized gluon is a major goal of the EIC it is interesting to study the sensitivity of jet production to this important quantity. The result obtained without the polarized gluon contribution differs by more than the estimated errors throughout the region $15 \mathrm{GeV} \leq p_{T}^{j} \leq 35 \mathrm{GeV}$. The shape of $A_{L L}$ as a function of $\eta^{j}$ is qualitatively different when the polarized gluon is turned off. Although the estimated statistical error indicates that this effect may be observable, the smallness of the asymmetry and the uncertainties in the resolved photon distribution indicate that such a determination of the polarized gluon may be difficult.

To illustrate the structure of the asymmetry we split it into partonic contributions in Fig. 9. In these plots we have

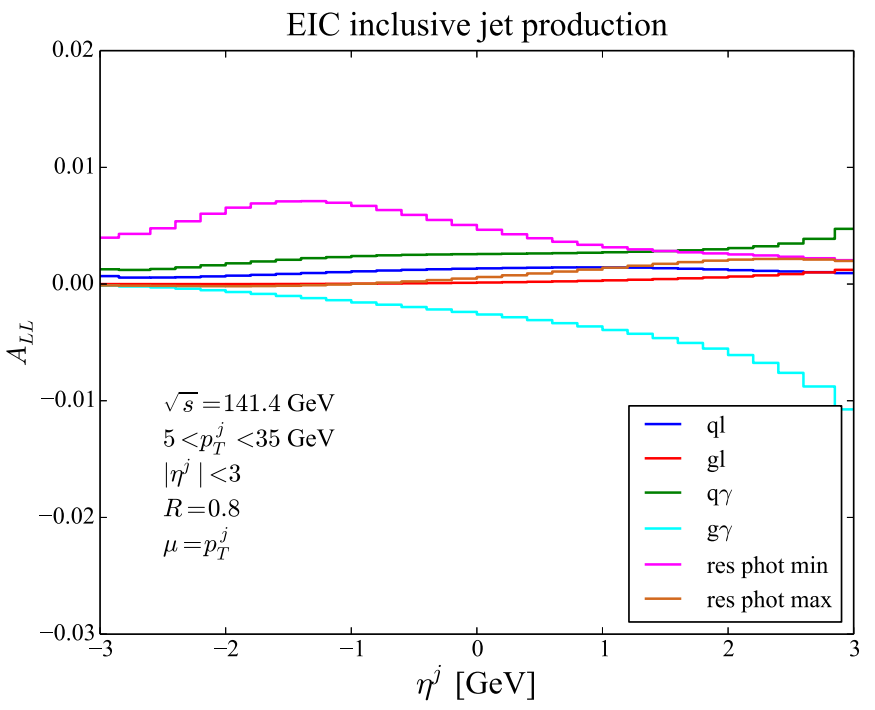

FIG. 9. Splits of the spin asymmetry as functions of the jet transverse momentum and pseudorapidity into partonic channels. 

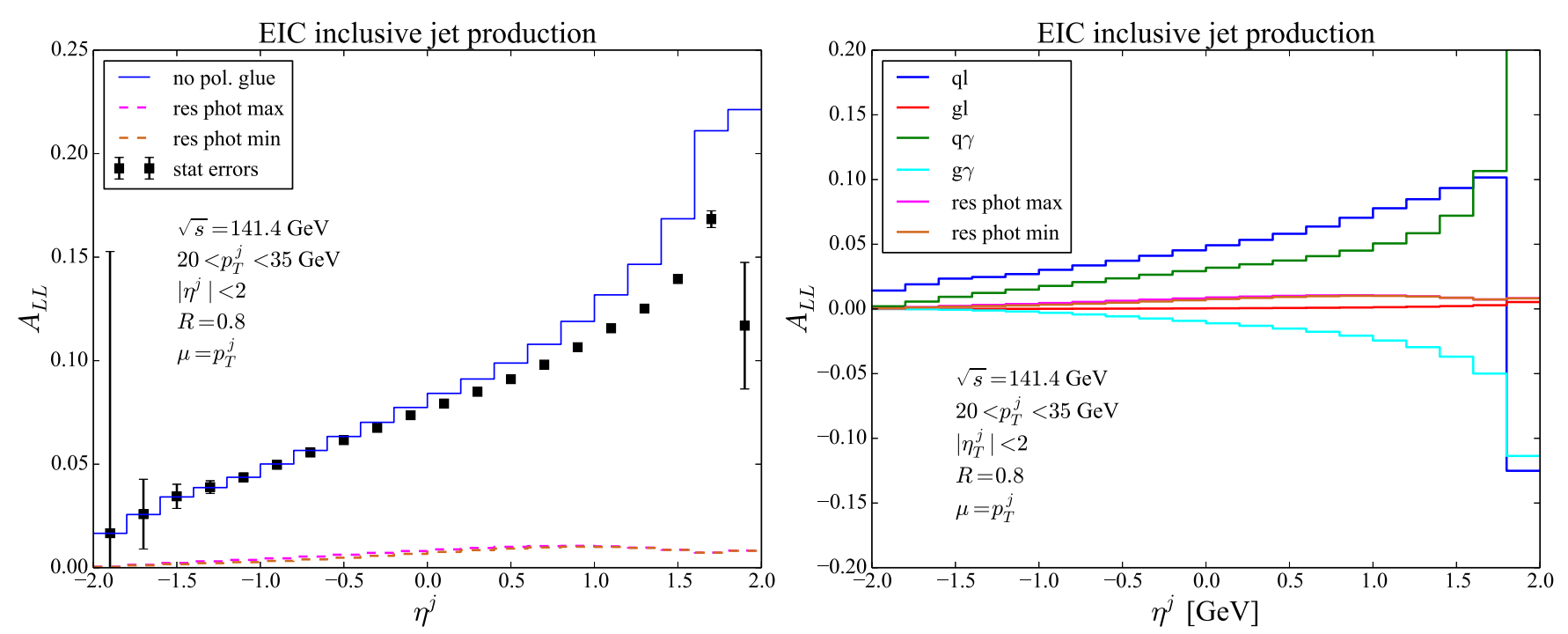

FIG. 10. Spin asymmetry as a function of $\eta^{j}$ with the cut $p_{T}^{j}>20 \mathrm{GeV}$.

kept only the indicated partonic channel in the numerator of the asymmetry, but all channels in the denominator. This makes each term directly proportional to the desired polarized distribution functions. The dominant contribution to the asymmetry at intermediate-to-high $p_{T}^{j}$ comes from the $q l$ channel. At intermediate $p_{T}^{j}$ the $q \gamma$ and $g \gamma$ contributions are important. It is interesting to note that the sensitivity to the polarized gluon distribution comes from the $g \gamma$ channel, which occurs only for inclusive jet production with $Q^{2} \approx 0$ without a tagged lepton. As we see explicitly later the sensitivity to $\Delta f_{g / H}$ vanishes upon imposing a large cut on $Q^{2}$. This demonstrates the importance of jet production measurements without a tagged lepton at a future EIC to give a direct determination of the polarized gluon distribution. The $A_{L L}$ distribution is dominated at low $\eta^{j}$ by the resolved photon term in the minimal model of this distribution, while at high $-\eta^{j}$ the $g \gamma$ channel determines the shape of the distribution. The resolved photon contribution is small if instead the maximal model of Ref. [34] is assumed. Again, this contribution only occurs for inclusive jet production without a tagged lepton.

In order to increase the sensitivity of $A_{L L}$ to the polarized gluon distribution, the previous results motivate isolating the high- $p_{T}^{j}$ region where Fig. 8 indicates that the contribution of this quantity becomes significant. We impose the more stringent cut $p_{T}^{j}>20 \mathrm{GeV}$ and show both the $A_{L L}$ distribution and its split into partonic channels in

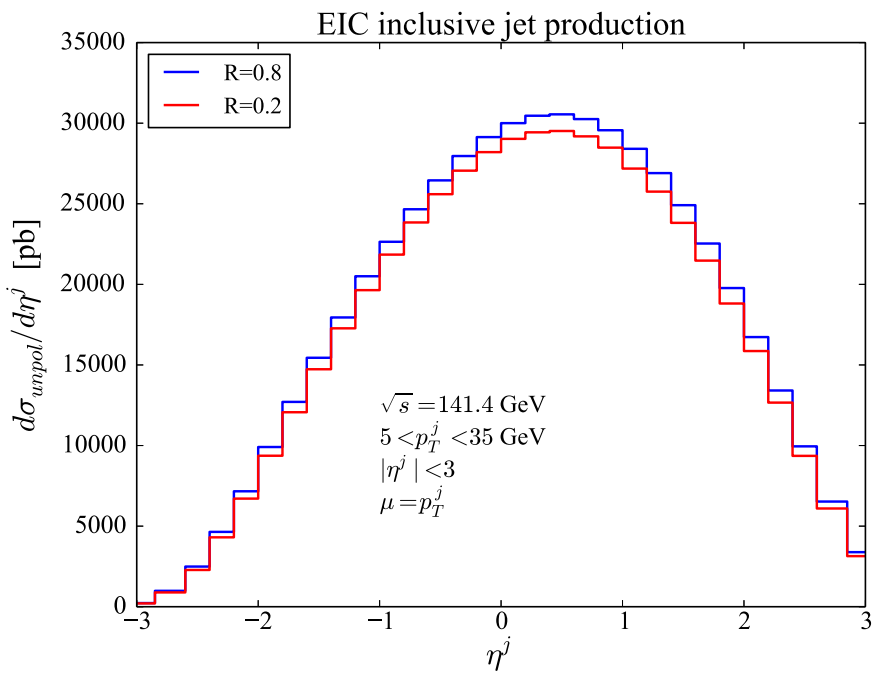

FIG. 11. Unpolarized cross section as a function of jet transverse momentum (left panel) and jet pseudorapidity (right panel) for the two jet radius parameters $R=0.2$ and $R=0.8$. 

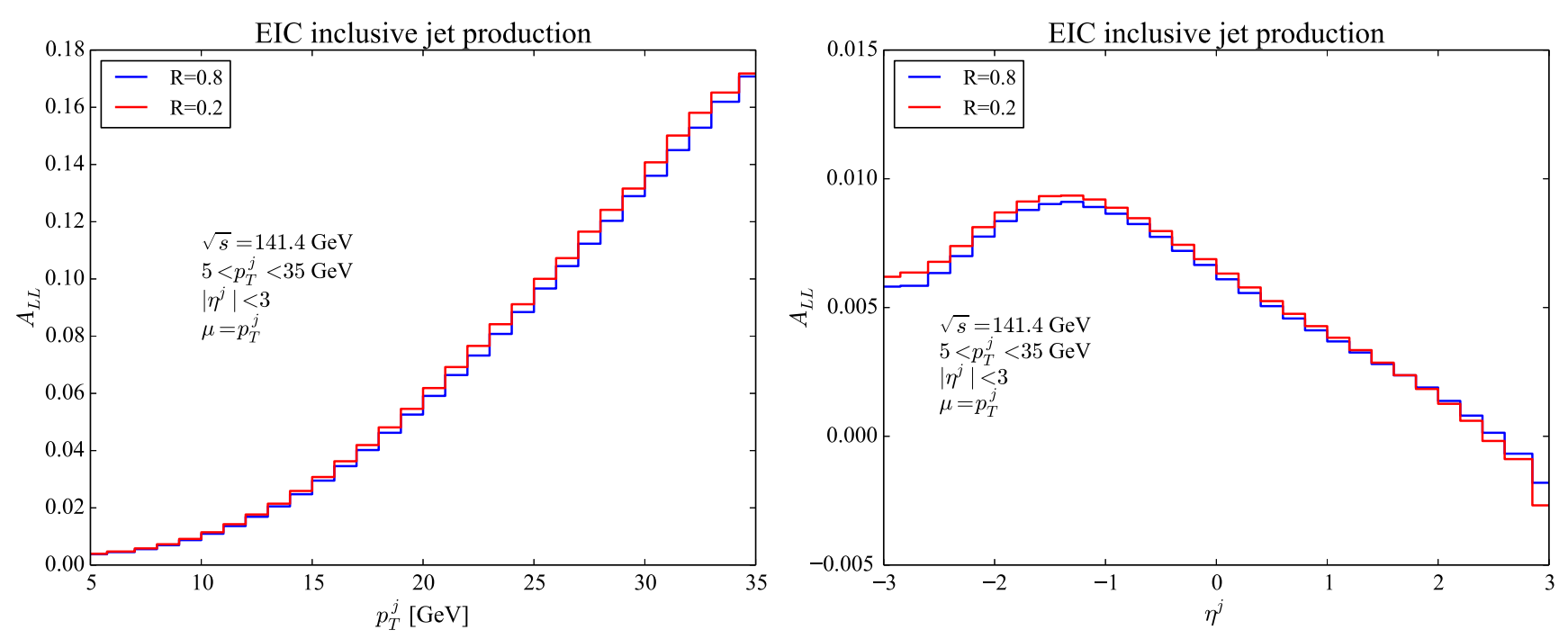

FIG. 12. Spin asymmetry as a function of jet transverse momentum (left panel) and jet pseudorapidity (right panel) for the two jet radius parameters $R=0.2$ and $R=0.8$.

Fig. 10. We note that the result without the polarized gluon differs significantly for positive $\eta^{j}$ from the one with the polarized gluon included. Both models for the polarized distribution of the photon are small for this $p_{T}^{j}$ cut, suggesting excellent sensitivity of this quantity to the polarized structure of the proton. The split into partonic channels shows that the dominant channels are the $q l, q \gamma$, and $g \gamma$ ones. We note a significant cancellation between the $q \gamma$ and $g \gamma$ channels that is relaxed when $\Delta f_{g / H}$ is turned off, leading to the larger asymmetry without the polarized gluon in the left plot of Fig. 10.

Since the NLO real-emission corrections to the $q l$ and $g l$ channels contain two final-state partons that can be arbitrarily separated in azimuthal angle and pseudorapidity

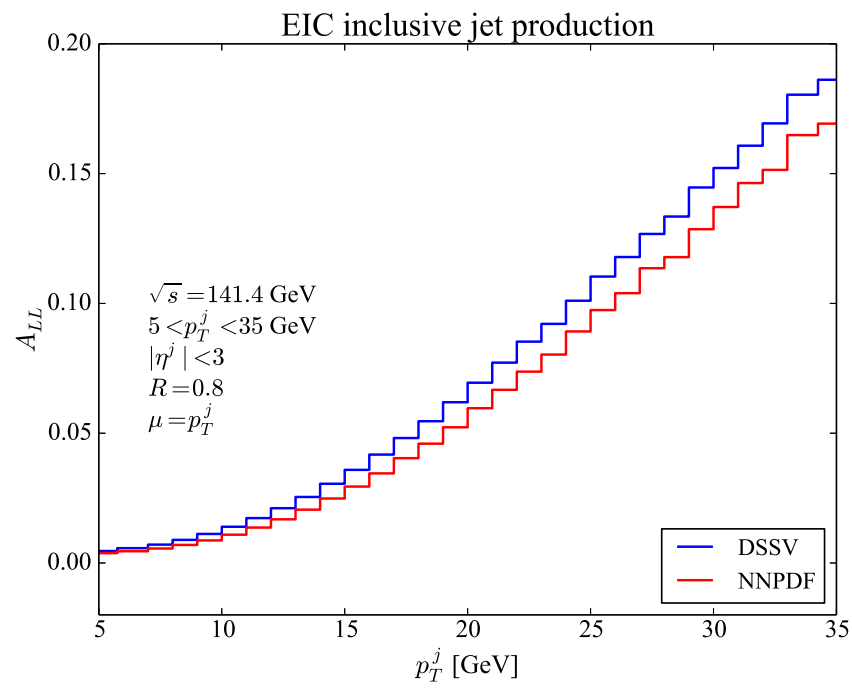

due to the presence of the wide-angle lepton, as can be seen from Fig. 2, there is a nontrivial dependence on the anti- $k_{T}$ radius parameter $R$ that begins at this order. In order to investigate the dependence on $R$ we study both the unpolarized cross section and $A_{L L}$ for the two choices $R=0.2$ and $R=0.8$ in Figs. 11 and 12 . We see that the dependence on the jet radius is minimal. The choice $R=0.8$ gives a slightly smaller cross section and a softer asymmetry as a function of $p_{T}^{j}$, but the effects on $A_{L L}$ are well within other theoretical uncertainties and the expected experimental errors. Given the small observed effect of changing $R$ in our fixed-order analysis, and also the fact that resummation effects would tend to cancel in the ratio of polarized and unpolarized cross sections needed for $A_{L L}$,

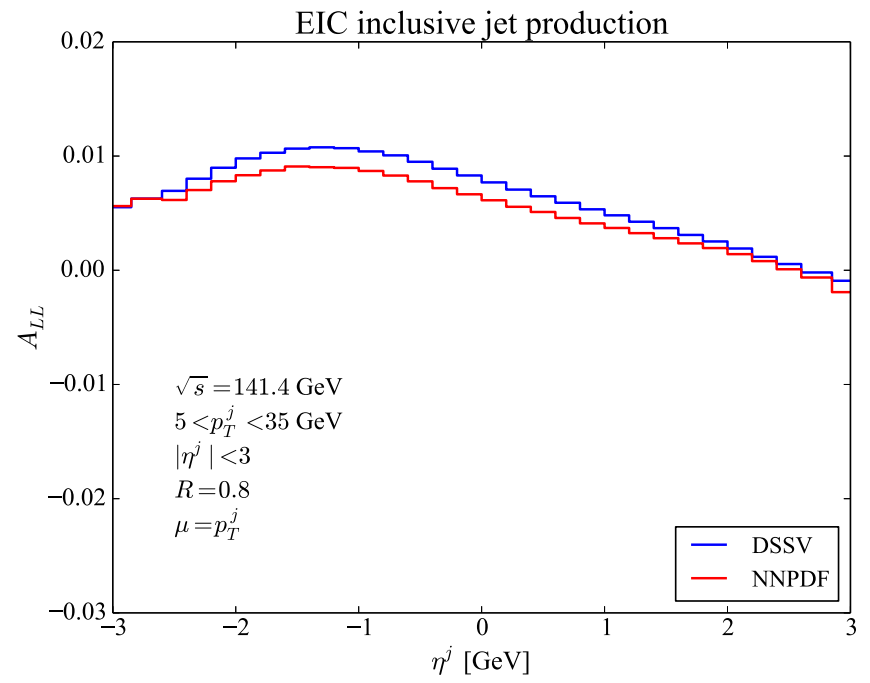

FIG. 13. Spin asymmetry as a function of jet transverse momentum (left panel) and jet pseudorapidity (right panel) for the NNPDF and DSSV extractions of polarized PDFs. 

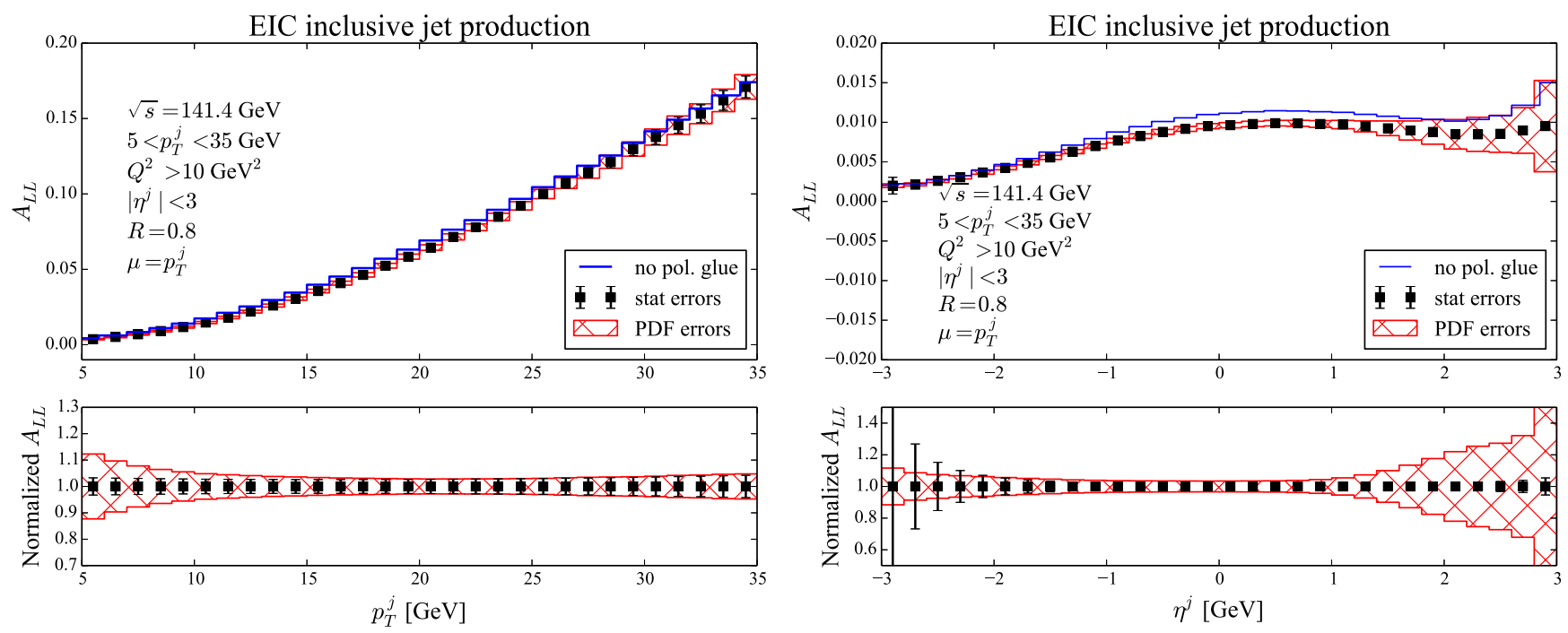

FIG. 14. Spin asymmetry as a function of jet transverse momentum (left panel) and jet pseudorapidity (right panel). The resolved photon contribution is shown separately in the upper panel of each plot. The lower panels normalize the results to the central values in order to more clearly illustrate the errors. The cut $Q^{2}>10 \mathrm{GeV}^{2}$ has been imposed.

we do not expect resummation of $R$-dependent logarithms to have a significant impact on our study.

Finally, although we have used the NNPDF 1.1 polarized distribution functions for the presented results, other parametrizations of these quantities are available in the literature. To test the sensitivity of the EIC to different PDF parametrizations we compare the $A_{L L}$ results obtained using NNPDF to those obtained using the DSSV global fit to the available data [6]. The $A_{L L}$ distribution as a function of both $p_{T}^{j}$ and $\eta^{j}$ is shown in Fig. 13 for the central values of both PDF sets. There are slight differences between the asymmetries obtained using the two different parametrizations. However, comparison with Fig. 8 reveals that all differences are well within the PDF uncertainties as estimated in the NNPDF fit.

\section{B. Jet production with a tagged lepton}

We next study the spin asymmetry when the cut $Q^{2}>$ $10 \mathrm{GeV}^{2}$ is imposed, indicating the presence of a wideangle lepton in the final state. As mentioned previously this cut significantly changes the structure of the cross section. The virtual photon emitted from the lepton is far off shell when this cut is imposed. Since the virtuality is much larger than the electron mass there are no longer contributions from the $q \gamma$ and $g \gamma$ channels in our theoretical framework, since these contributions become a useful description only

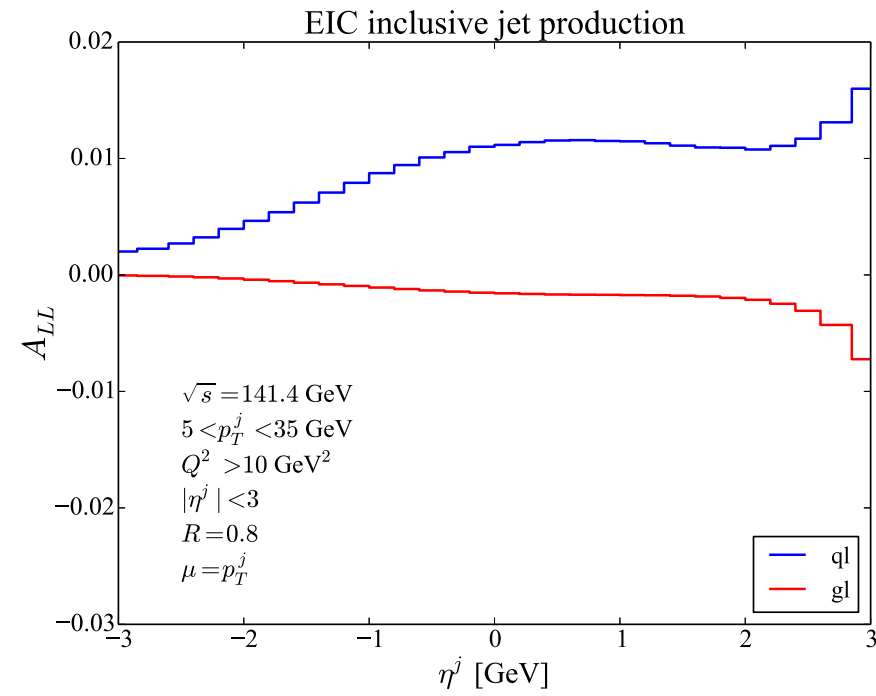

FIG. 15. Splits of the spin asymmetry as functions of the jet transverse momentum and pseudorapidity into partonic channels. The cut $Q^{2}>10 \mathrm{GeV}^{2}$ has been imposed. 
when $Q^{2} \lesssim m_{\text {elec }}^{2}$. Similarly, since $Q^{2} \gg \Lambda_{\mathrm{OCD}}^{2}$ there are no longer resolved photon contributions. Splitting of the photon into partons at large $Q^{2}$ happens perturbatively without large logarithmic contributions at higher orders in $\alpha_{s}$ than those considered in this study, and are not enhanced. This leaves only the $q l$ and $g l$ channels to consider.

The results for the distributions of $A_{L L}$ in $p_{T}^{j}$ and $\eta^{j}$ are shown in Fig. 14, while the splits into the $q l$ and $g l$ channels are shown in Fig. 15. We note that the PDF uncertainties are smaller than for inclusive jet production, particularly at low $p_{T}^{j}$ and high $\eta^{j}$. These regions receive significant contributions from the $g \gamma$ channel in the inclusive jet production case but not here, indicating the reason for this difference. The $q l$ channel dominates for all values of transverse momentum, and for all but the very forward region of pseudorapidity. This reveals the advantage of inclusive jet measurements compared to the process of jet production with a tagged lepton from the perspective of constraining the polarized gluon distribution of the proton.

\section{RESULTS FOR $\sqrt{s}=63.2 \mathrm{GeV}$}

We now study jet production at an EIC with center-ofmass energy $\sqrt{s}=63.2 \mathrm{GeV}$. Due to the reduced collision energy the accessible kinematic range of the jets is reduced,
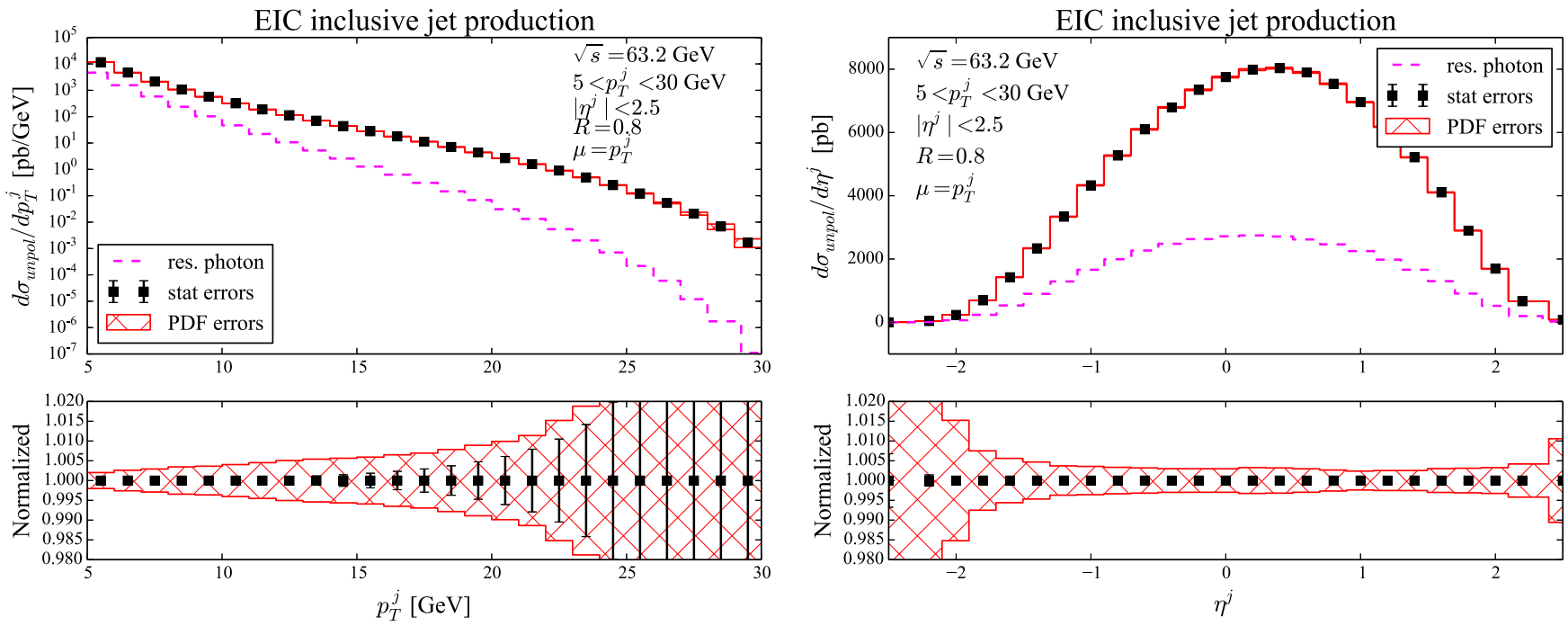

FIG. 16. Total unpolarized cross section as a function of jet transverse momentum (left panel) and jet pseudorapidity (right panel) for $\sqrt{s}=63.2 \mathrm{GeV}$. The resolved photon contribution is shown separately in the upper panel of each plot. The lower panels normalize the results to the central values in order to more clearly illustrate the errors.
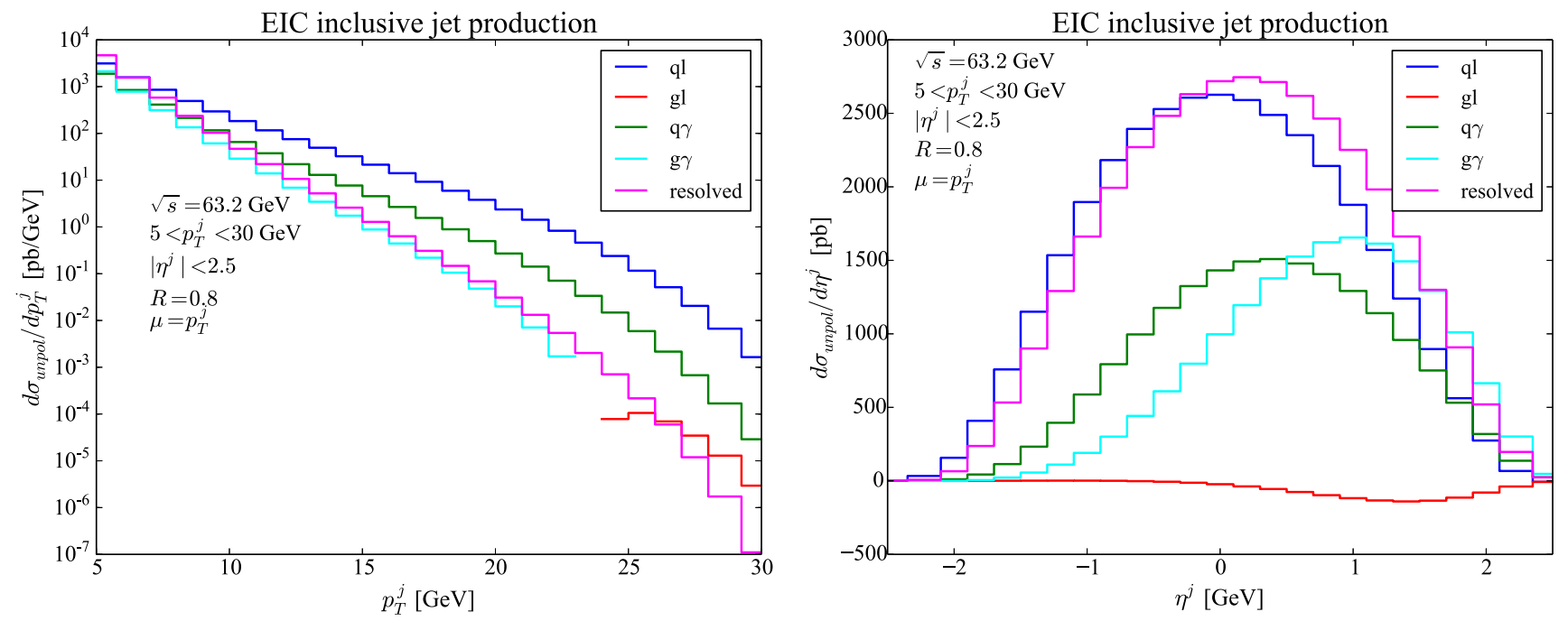

FIG. 17. Split of the unpolarized transverse momentum and pseudorapidity distributions for $\sqrt{s}=63.2$ GeV into partonic channels as described in the text. 
and we consequently focus on the phase-space region $5<p_{T}^{j}<30 \mathrm{GeV}$ and $\left|\eta^{j}\right|<2.5$. The collisions at this energy typically occur at higher Bjorken- $x$, and this determines much of the observed phenomenology.

We show first in Fig. 16 the unpolarized cross section as a function of both $p_{T}^{j}$ and $\eta^{j}$. The corresponding split into partonic channels is shown in Fig. 17. Although still the largest partonic channel at low $p_{T}^{j}$, the resolved photon contribution is no longer a factor of several larger than the other channels, unlike for $\sqrt{s}=141.4 \mathrm{GeV}$. This is because the parton-in-lepton distributions required to obtain these channels fall off more rapidly with Bjorken- $x$ due to the multiple collinear splittings needed to generate them. This can be observed from the additional convolution in Eq. (7). Above $p_{T}^{j} \approx 10 \mathrm{GeV}$ the $q l$ channel dominates, with the second largest being the $q \gamma$ channel. All channels contribute non-negligibly to the $\eta^{j}$ distribution except $g l$, which is small throughout phase space. We note that the $g l$ channel is small and negative for small $p_{T}^{j}$, which explains why it begins at $p_{T}^{j} \approx 25 \mathrm{GeV}$ in the left panel of Fig. 17. This channel by itself is not a physical observable, but is only a component of the full NLO cross section defined in the $\overline{\mathrm{MS}}$ scheme. We note that the PDF errors are larger at high $p_{T}^{j}$ than the
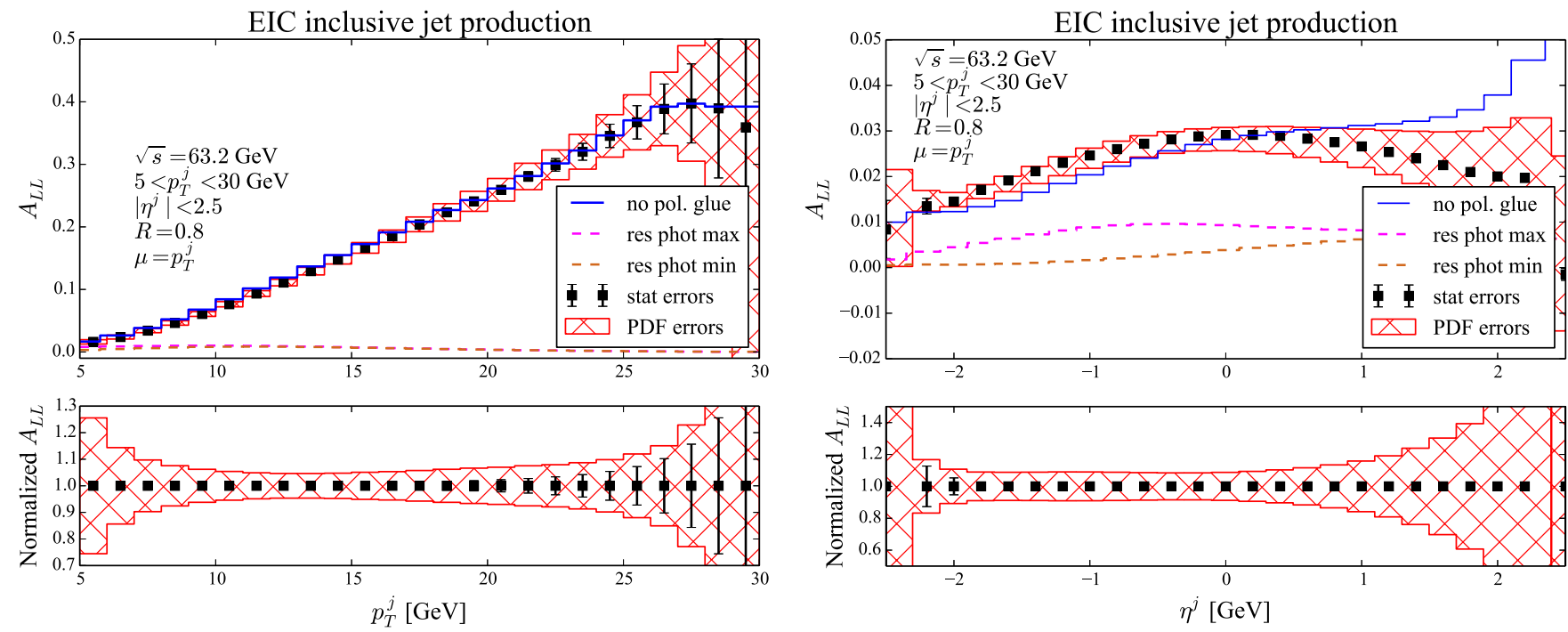

FIG. 18. Spin asymmetry as a function of jet transverse momentum (left panel) and jet pseudorapidity (right panel) for $\sqrt{s}=63.2 \mathrm{GeV}$. The resolved photon contribution is shown separately in the upper panel of each plot. The lower panels normalize the results to the central values in order to more clearly illustrate the errors.
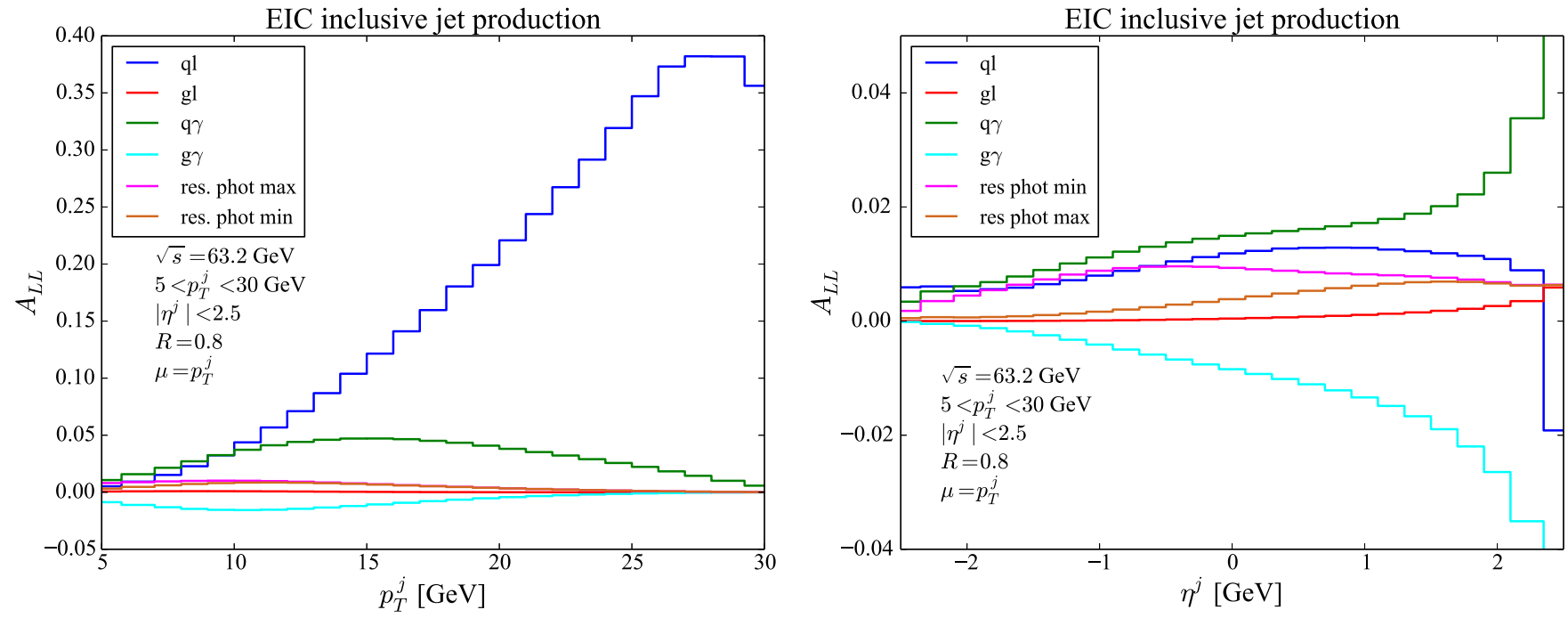

FIG. 19. Splits of the spin asymmetry as functions of the jet transverse momentum and pseudorapidity for $\sqrt{s}=63.2 \mathrm{GeV}$ into partonic channels. 
corresponding errors for $\sqrt{s}=141.4 \mathrm{GeV}$. Ths is because the PDFs are being probed at very high Bjorken- $x$ where constraints from current data are limited. However, the estimated statistical errors also become large in this region.

We now study the spin asymmetry for $\sqrt{s}=63.2 \mathrm{GeV}$. The distributions of $A_{L L}$ in both jet transverse momentum and pseudorapidity are shown in Fig. 18. The separate partonic channels are shown in Fig. 19. As before the separate contributions of both polarized resolved photon models are shown, as well as the result obtained by turning off the polarized gluon distribution. The asymmetries are larger than observed for $\sqrt{s}=141.4 \mathrm{GeV}$, reaching $40 \%$ at large $p_{T}^{j}$ and nearly $3 \%$ for central $\eta^{j}$ when integrated over transverse momentum. The $q l$ channel dominates the asymmetry as a function of $p_{T}^{j}$ for all but the lowest few bins, where other partonic channels such as the resolved photon contributions, the $q \gamma$, and the $g \gamma$ terms all become important. While the contribution of the $q l$ channel can be reduced by focusing on the low transverse momentum range $p_{T}^{j} \lesssim 10 \mathrm{GeV}$, both the polarized gluon and resolved photon distributions become important for this region. This is different than for $\sqrt{s}=141.4 \mathrm{GeV}$, where the polarized gluon contributions could be more easily isolated.
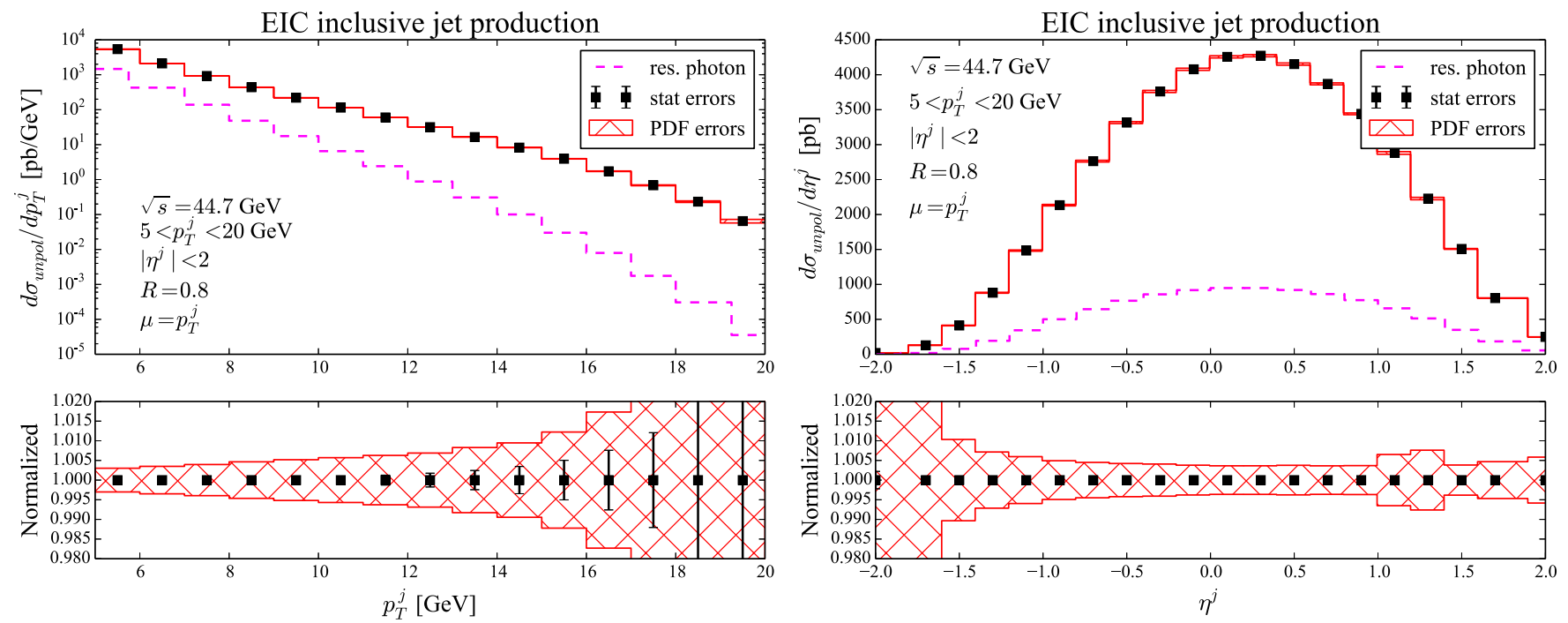

FIG. 20. Total unpolarized cross section as a function of jet transverse momentum (left panel) and jet pseudorapidity (right panel) for $\sqrt{s}=44.7 \mathrm{GeV}$. The resolved photon contribution is shown separately in the upper panel of each plot. The lower panels normalize the results to the central values in order to more clearly illustrate the errors.
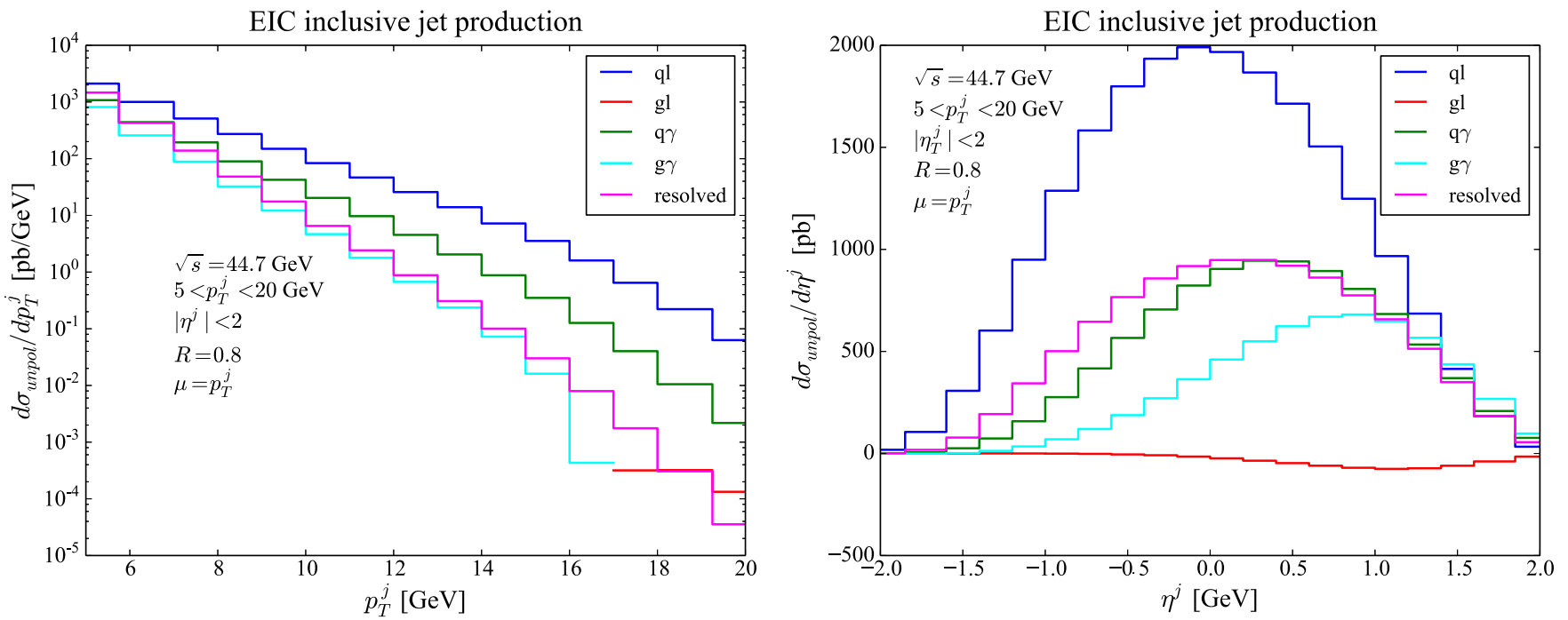

FIG. 21. Split of the unpolarized transverse momentum and pseudorapidity distributions for $\sqrt{s}=44.7 \mathrm{GeV}$ into partonic channels as described in the text. 
The $\eta^{j}$ distribution again shows a difference in shape between the minimal and maximal models of the polarized photon distributions, although this channel is smaller than the $q l$ and $q \gamma$ ones throughout most of the phase space. The forward $\eta^{j}$ region exhibits a strong interplay between the $q \gamma$ and $g \gamma$ channels. They significantly cancel, indicating sensitivity to modifications of the polarized gluon distribution. However, we note that the size of the $A_{L L}$ contribution coming from the $g \gamma$ channel is significantly smaller than observed for $\sqrt{s}=141.4 \mathrm{GeV}$ with $p_{T}^{j}>20 \mathrm{GeV}$ in Fig. 10, while the relative contribution from the resolved photon channel is larger, suggesting less sensitivity to this quantity than for the larger energy collisions.

\section{RESULTS FOR $\sqrt{s}=\mathbf{4 4 . 7} \mathrm{GeV}$}

Finally, we focus on our lowest considered center-ofmass scattering energy, $\sqrt{s}=44.7 \mathrm{GeV}$. We consider the kinematic range $5<p_{T}^{j}<20 \mathrm{GeV}$ and $\left|\eta^{j}\right|<2$. We first show in Fig. 20 the total cross section as a function of transverse momentum and pseudorapidity. The splits of these distributions into separate partonic channels are shown in Fig. 21. The most prominent difference with respect to the previously studied energies is that the resolved photon contributions are no longer dominant in any region of phase space. The $q l$ channel is the largest over the entire studied region, followed by the $q \gamma$ channel.
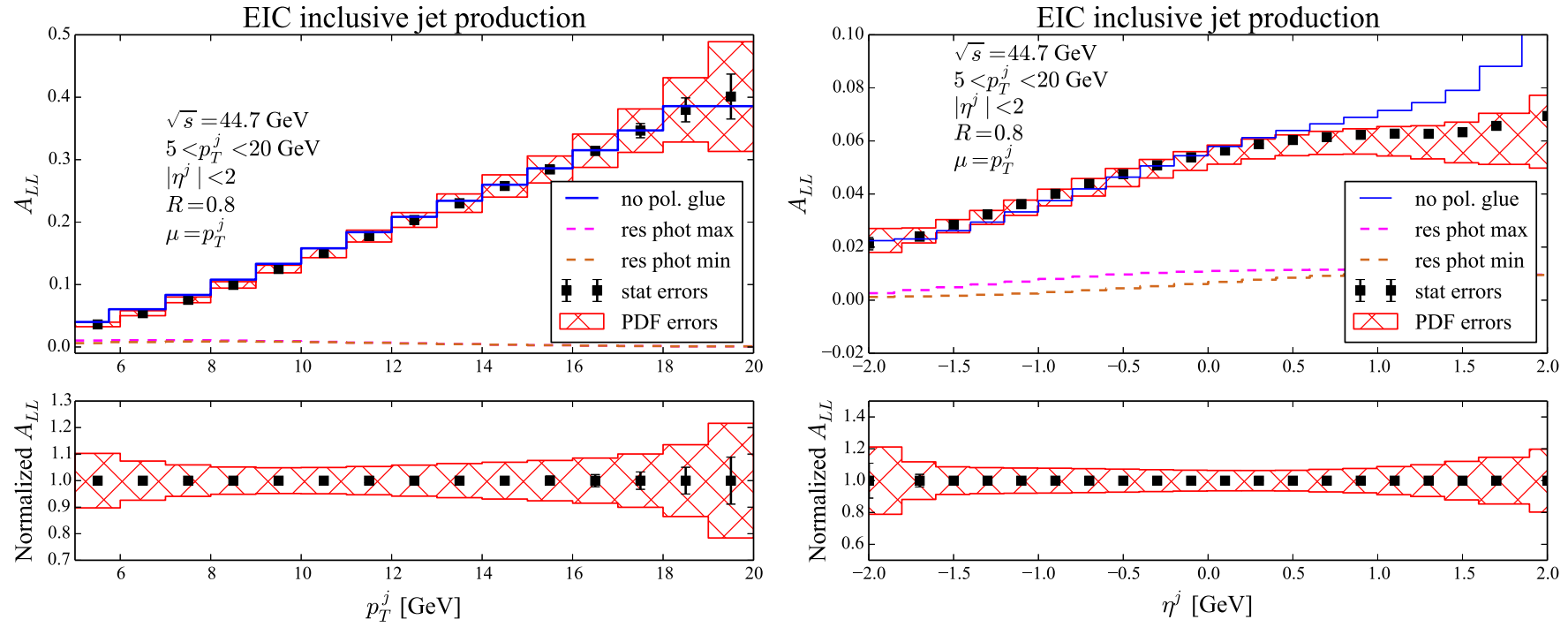

FIG. 22. Spin asymmetry as a function of jet transverse momentum (left panel) and jet pseudorapidity (right panel) for $\sqrt{s}=44.7 \mathrm{GeV}$. The resolved photon contribution is shown separately in the upper panel of each plot. The lower panels normalize the results to the central values in order to more clearly illustrate the errors.
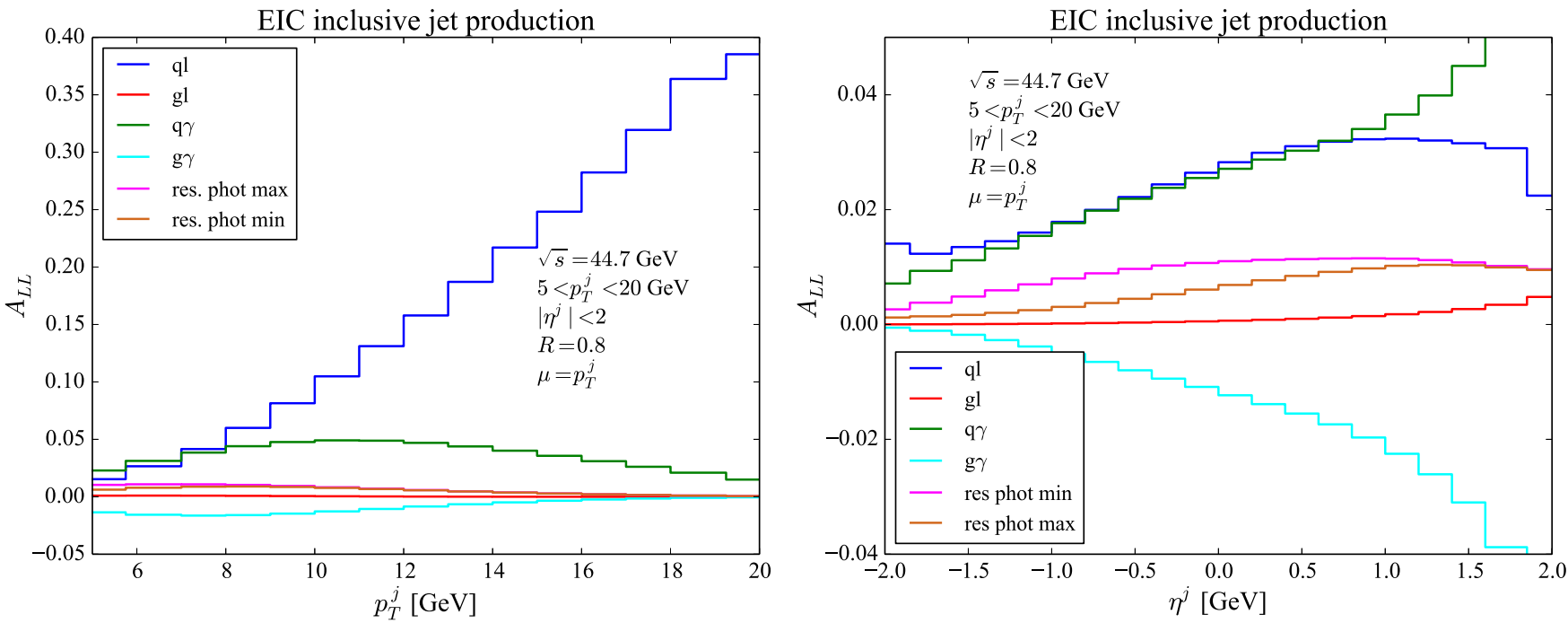

FIG. 23. Splits of the spin asymmetry as functions of the jet transverse momentum and pseudorapidity for $\sqrt{s}=44.7 \mathrm{GeV}$ into partonic channels. 
From the perspective of better determining the partonic structure of the photon via jet production, it is advantageous to be at higher collision energies.

In Fig. 22 we show the spin asymmetry $A_{L L}$ as a function of $p_{T}^{j}$ and $\eta^{j}$. The contributions of the separate partonic channels are shown in Fig. 23. We first note that the estimated PDF errors are much smaller than for larger center-of-mass energies, reaching only a maximum of $20 \%$ at the boundaries of phase space. This is in contrast to $\sqrt{s}=141.4 \mathrm{GeV}$, where Fig. 8 exhibits PDF errors reaching $40 \%$ or more for $\eta^{j}>0$ and $p_{T}^{j}<10 \mathrm{GeV}$. As Fig. 5 makes clear, collisions at $\sqrt{s}=44.7 \mathrm{GeV}$ probe large Bjorken- $x$ where some knowledge of the polarized structure of the proton is available. Higher collision energies probe smaller $x$, which are still undetermined from data. Broader coverage of the polarized PDFs is obtained by measurements at higher scattering energy. While there are differences between the various models for the polarized resolved photons, both models give significantly smaller contributions than the $q l$ channel throughout phase space. There is a significant cancellation between the $q \gamma$ and $g \gamma$ contributions to the asymmetry in the forward $\eta^{j}$ region, but these separate contributions to the asymmetry only reach a few percent, smaller than observed for $\sqrt{s}=141.4 \mathrm{GeV}$ with the cut $p_{T}^{j}>20 \mathrm{GeV}$.

\section{SUMMARY AND CONCLUSIONS}

In this manuscript we presented a detailed phenomenological study of inclusive jet production at a future EIC. Our goal was to establish the ability of this process to probe the polarized structure of the proton, and to determine which kinematic regions of jet production are sensitive to different aspects of proton structure. We have considered several possibilities for the center-of-mass scattering energy of the proposed machine to elucidate how different EIC realizations can improve our knowledge of proton structure. We have studied the effects of different PDF parametrizations, finite jet radii and the effect of tagging the final-state lepton. Both the polarized proton and photon structures were considered in our analysis. The effects of statistical and PDF uncertainties were studied in detail.

Our study was performed using fixed-order perturbative QCD through NLO with all relevant partonic processes included, including the resolved contributions associated with the nonperturbative structure of the photon. The entire theoretical framework for both polarized and unpolarized collisions has been implemented in the flexible numerical code DISTRESS designed for phenomenological studies at a future EIC. The major findings of our study are summarized below.

(i) Collisions at the highest considered center-of-mass energy, $\sqrt{s}=141.4 \mathrm{GeV}$, offer the broadest sensitivity to polarized hadronic structure. Both the resolved photon distributions and the polarized gluons and quarks can be probed by selecting appropriate regions of jet transverse momentum and pseudorapidity. Low transverse momenta provide access to the resolved photon, while intermediate-to-high transverse momenta are sensitive to the polarized gluon. As the scattering energy is decreased the sensitivity to the polarized photon distributions decreases, since these distributions fall rapidly as higher Bjorken- $x$ values are probed in lower-energy collisions.

(ii) Generically, across all collision energies, the estimated polarized PDF errors are larger than the expected EIC statistical errors, which indicates that all collision energies can potentially provide useful information in global fits to constrain polarized PDFs. Previous work [25] has shown that the theoretical scale uncertainties are small once NNLO corrections are included. There is an excellent chance to learn more about polarized proton structure at the EIC.

(iii) The sensitivity to the polarized gluon comes from scattering channels such as $g \gamma$ which become active when $Q^{2} \approx 0$. Large polarized quark contributions with significant PDF errors come from the $q l$ scattering channel at high transverse momentum. Resolved photon distributions can be accessed at low transverse momentum. This shows the importance of inclusive jet production, since it gives handles on all relevant distributions in different kinematic regions.

\section{ACKNOWLEDGMENTS}

We thank J. Qiu, B. Page and W. Vogelsang for comments on the manuscript. R. B. is supported by the DOE Award No. DE-AC02-06CH11357. F. P. is supported by the DOE Awards No. DE-FG02-91ER40684 and No. DE$\mathrm{AC} 02-06 \mathrm{CH} 11357$. H.X. is supported by the DOE Award No. DE-AC02-06CH11357 and the NSF Grant No. NSF-1740142. This research used resources of the Argonne Leadership Computing Facility, which is a DOE Office of Science User Facility supported under Contract No. DE-AC02-06CH11357. 
[1] J. Ashman et al. (European Muon Collaboration), Phys. Lett. B 206, 364 (1988).

[2] J. Ashman et al. (European Muon Collaboration), Nucl. Phys. B328, 1 (1989).

[3] A. Accardi et al., Eur. Phys. J. A 52, 268 (2016).

[4] X. D. Ji, Phys. Rev. Lett. 78, 610 (1997).

[5] R. L. Jaffe and A. Manohar, Nucl. Phys. B337, 509 (1990).

[6] D. de Florian, R. Sassot, M. Stratmann, and W. Vogelsang, Phys. Rev. Lett. 113, 012001 (2014).

[7] E. R. Nocera, R. D. Ball, S. Forte, G. Ridolfi, and J. Rojo (NNPDF Collaboration), Nucl. Phys. B887, 276 (2014).

[8] L. A. Harland-Lang, A. D. Martin, P. Motylinski, and R. S. Thorne, Eur. Phys. J. C 75, 204 (2015).

[9] S. Dulat, T.-J. Hou, J. Gao, M. Guzzi, J. Huston, P. Nadolsky, J. Pumplin, C. Schmidt, D. Stump, and C.-P. Yuan, Phys. Rev. D 93, 033006 (2016).

[10] R. D. Ball et al. (NNPDF Collaboration), Eur. Phys. J. C 77, 663 (2017).

[11] M. Klasen, K. Kovarik, and J. Potthoff, Phys. Rev. D 95, 094013 (2017).

[12] M. Klasen and K. Kovak, Phys. Rev. D 97, 114013 (2018).

[13] Z. B. Kang, A. Metz, J. W. Qiu, and J. Zhou, Phys. Rev. D 84, 034046 (2011).

[14] P. Hinderer, M. Schlegel, and W. Vogelsang, Phys. Rev. D 96, 014002 (2017).

[15] C. Uebler, A. Schfer, and W. Vogelsang, Phys. Rev. D 96, 074026 (2017).

[16] B. Jager, M. Stratmann, and W. Vogelsang, Phys. Rev. D 70, 034010 (2004).

[17] D. de Florian and S. Frixione, Phys. Lett. B 457, 236 (1999).

[18] B. Jager, Phys. Rev. D 78, 034017 (2008).

[19] R. Boughezal, C. Focke, X. Liu, and F. Petriello, Phys. Rev. Lett. 115, 062002 (2015).

[20] J. Gaunt, M. Stahlhofen, F. J. Tackmann, and J. R. Walsh, J. High Energy Phys. 09 (2015) 058.
[21] R. Boughezal, F. Petriello, U. Schubert, and H. Xing, Phys. Rev. D 96, 034001 (2017).

[22] E. C. Aschenauer, R. Sassot, and M. Stratmann, Phys. Rev. D 92, 094030 (2015).

[23] X. Chu, E. C. Aschenauer, J.H. Lee, and L. Zheng, Phys. Rev. D 96, 074035 (2017).

[24] E. C. Aschenauer, S. Fazio, M. A. C. Lamont, H. Paukkunen, and P. Zurita, Phys. Rev. D 96, 114005 (2017).

[25] G. Abelof, R. Boughezal, X. Liu, and F. Petriello, Phys. Lett. B 763, 52 (2016).

[26] S. Catani and M.H. Seymour, Nucl. Phys. B485, 291 (1997); B510, 503(E) (1998).

[27] P. Hinderer, M. Schlegel, and W. Vogelsang, Phys. Rev. D 92, 014001 (2015); 93, 119903(E) (2016).

[28] I. W. Stewart, F. J. Tackmann, and W. J. Waalewijn, Phys. Rev. Lett. 105, 092002 (2010).

[29] D. Kang, C. Lee, and I. W. Stewart, Phys. Rev. D 88, 054004 (2013).

[30] I. W. Stewart, F. J. Tackmann, and W. J. Waalewijn, Phys. Rev. D 81, 094035 (2010).

[31] I. W. Stewart, F. J. Tackmann, and W. J. Waalewijn, Phys. Rev. Lett. 106, 032001 (2011).

[32] Z. B. Kang, S. Mantry, and J. W. Qiu, Phys. Rev. D 86, 114011 (2012).

[33] Z. B. Kang, X. Liu, and S. Mantry, Phys. Rev. D 90, 014041 (2014).

[34] M. Gluck and W. Vogelsang, Z. Phys. C 55, 353 (1992).

[35] M. Klasen, Rev. Mod. Phys. 74, 1221 (2002).

[36] M. Cacciari, G. P. Salam, and G. Soyez, J. High Energy Phys. 04 (2008) 063.

[37] R. D. Ball L. Del Debbio, S. Forte, A. Guffanti, J. I. Latorre, A. Piccione, J. Rojo, and M. Ubiali (NNPDF Collaboration), Nucl. Phys. B809, 1 (2009); B816, 293(E) (2009).

[38] M. Gluck, E. Reya, and A. Vogt, Phys. Rev. D 46, 1973 (1992).

[39] E. C. Aschenauer et al., arXiv:1708.01527. 